Centre interuniversitaire de recherche

en économie quantitative

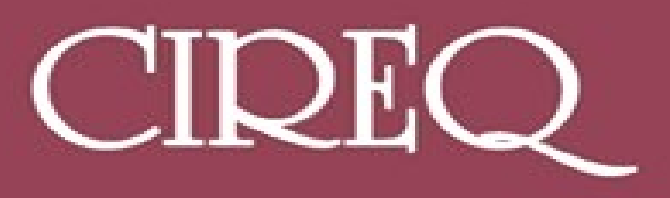

Cahier 03-2012

Top Trading with Fixed Tie-Breaking in Markets with Indivisible Goods

LarS EHLERS 


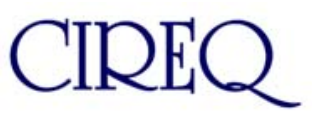

Le Centre interuniversitaire de recherche en économie quantitative (CIREQ) regroupe des chercheurs dans les domaines de l'économétrie, la théorie de la décision, la macroéconomie et les marchés financiers, la microéconomie appliquée et l'économie expérimentale ainsi que l'économie de l'environnement et des ressources naturelles. Ils proviennent principalement des universités de Montréal, McGill et Concordia. Le CIREQ offre un milieu dynamique de recherche en économie quantitative grâce au grand nombre d'activités qu'il organise (séminaires, ateliers, colloques) et de collaborateurs qu'il reçoit chaque année.

The Center for Interuniversity Research in Quantitative Economics (CIREQ) regroups researchers in the fields of econometrics, decision theory, macroeconomics and financial markets, applied microeconomics and experimental economics, and environmental and natural resources economics. They come mainly from the Université de Montréal, McGill University and Concordia University. CIREQ offers a dynamic environment of research in quantitative economics thanks to the large number of activities that it organizes (seminars, workshops, conferences) and to the visitors it receives every year.

\title{
Cahier 03-2012
}

\section{Top Trading with Fixed Tie-Breaking in Markets with Indivisible Goods}

\author{
LarS EHLERS
}

CIREQ, Université de Montréal C.P. 6128, succursale Centre-ville Montréal (Québec) H3C 3J7 Canada
Université th de Montréal
McGill

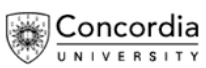


Ce cahier a également été publié par le Département de sciences économiques de l'Université de Montréal sous le numéro (2012-02).

This working paper was also published by the Department of Economics of the University of Montreal under number (2012-02).

Dépôt légal - Bibliothèque nationale du Canada, 2012, ISSN 0821-4441

Dépôt légal - Bibliothèque et Archives nationales du Québec, 2012 ISBN-13 : 978-2-89382-626-4 


\title{
Top Trading with Fixed Tie-Breaking in Markets with Indivisible Goods*
}

\author{
Lars Ehlers ${ }^{\dagger}$
}

March 2012

\begin{abstract}
We study markets with indivisible goods where monetary compensations are not possible. Each individual is endowed with an object and a preference relation over all objects. When preferences are strict, Gale's top trading cycle algorithm finds the unique core allocation. When preferences are not necessarily strict, we use an exogenous profile of tie-breakers to resolve any ties in individuals' preferences and apply Gale's top trading cycle algorithm for the resulting profile of strict preferences. We provide a foundation of these simple extensions of Gale's top trading cycle algorithm from strict preferences to weak preferences. We show that Gale's top trading cycle algorithm with fixed tie-breaking is characterized by individual rationality, strategy-proofness, weak efficiency, non-bossiness, and consistency. Our result supports the common practice in applications to break ties in weak preferences using some fixed exogenous criteria and then to use a "good and simple" rule for the resulting strict preferences. This reinforces the market-based approach even in the presence of indifferences because always competitive allocations are chosen.
\end{abstract}

JEL Classification: C78, D63, D70

Keywords: indivisible goods, top trading cycles, indifferences, fixed tie-breaking.

\section{Introduction}

Consider the problem of exchanging a number of indivisible goods ("houses" or "objects") among a group of individuals without monetary compensations. Each individual is endowed with one object and consumes exactly one object. The individuals do not pay for the objects; neither is any form of side payments between the individuals permitted. Clearly, this type of problem arises when reallocating courses among professors, apartments among existing tenants (where rents are

\footnotetext{
*I am grateful to William Thomson, Tommy Andersson, Vikram Manjunath and Lars-Gunnar Svensson for their helpful comments and suggestions and to Lund University for its hospitality. I acknowledge financial support from the FQRSC (Québec), the SSHRC (Canada), and the Jan Wallander and Tom Hedelius Foundation.

${ }^{\dagger}$ Département de Sciences Économiques and CIREQ, Université de Montréal, Montréal, Québec H3C 3J7, Canada; e-mail: lars.ehlers@umontreal.ca.
} 
regulated), offices among employees, tasks among workers, seats at public schools among students, and time sharing slots of vacation apartments among their owners. ${ }^{1}$

The classical piece by Shapley and Scarf (1974) introduced this problem and uses the notion of Edgeworthian exchange to solve it. The individuals are free to engage in multilateral negotiations and exchange by using their allocation either individually or as part of a coalition to block proposed allocations. The set of unblocked allocations is the core. In their classic paper Shapley and Scarf (1974) use Gale's "Top Trading Cycle" algorithm to establish the existence of the core of this "housing market" (or market with indivisible goods). A number of subsequent "early" papers have used this "exchange based" approach to investigate further the properties of cores of markets with indivisibilities (see Roth and Postlewaite (1977), Wako (1984), and Ma (1994)) showing that in the special case when preferences are restricted to be linear orderings, the core exists, is unique and efficient, and corresponds to the unique competitive allocation of the housing market.

Recent literature over the past ten years regarded this problem where the indivisible goods are commonly owned and no individual has a priori any right for any available object. ${ }^{2}$ Then all individuals have equal right to consume any of the houses. Several characterizations of certain classes of mechanisms were obtained. All these mechanisms satisfy two basic properties, namely strategyproofness meaning that no individual can gain by misreporting his preference and non-bossiness (Satterthwaite and Sonnenschein, 1981) meaning that no individual can change the allocation without changing his assigned house. Using these two properties, Svensson (1999) characterized serial dictatorships with "neutrality", Pápai (2000) characterized hierarchical exchange rules with efficiency and "reallocation-proofness", and Pycia and Ünver (2009) characterized "trading cycles with brokers and owners" with efficiency. The last two characterizations used in addition efficiency whereas in the first one efficiency is a consequence of the other properties. Except for Svensson's characterization, the other two results characterize large classes of mechanisms. ${ }^{3}$ It is also well known that in house allocation with strict preferences, strategy-proofness and non-bossiness are equivalent to group strategy-proofness (Pápai, 2000). Group strategy-proofness means that no group of agents is able to manipulate the mechanism such that all members of the group become weakly better off and some are strictly better off.

In most of the literature on house allocation, agents are assumed to possess asymmetric, also called strict, preferences over the set of houses. In applications we cannot rule out that agents may be indifferent between various objects. There are several reasons why this might occur. For example, agents might have little information about the objects. Then we expect that they might

\footnotetext{
${ }^{1}$ The introduction in Pápai (2000) gives examples of other similar situations.

${ }^{2}$ For a survey, see Sönmez and Ünver (2009).

${ }^{3}$ Among others, Ergin (2002) and Ehlers and Klaus (2007) characterize mixed dictator-pairwise exchange rules. Again this class of rules satisfies strategy-proofness, non-bossiness, and efficiency. Furthermore, these properties are satisfied by the class of rules characterized by Sönmez and Ünver (2010): in house allocation with existing tenants they provide a foundation of the you request my house-I get your turn mechanisms introduced by Abdulkadiroglu and Sönmez (1999).
} 
not have the ability to distinguish among all objects. It is important to understand this case. In contrast with the characterizations of large classes of rules for the strict domain, when indifferences are allowed the results are less appealing. Ehlers (2002) shows that group strategy-proofness and efficiency are incompatible on the weak domain whereas Bogomolnaia, Deb and Ehlers (2005) show that basically only serial dictatorships ${ }^{4}$ satisfy strategy-proofness, non-bossiness, and efficiency. Therefore, for the weak domain the equivalence of strategy-proofness and non-bossiness with group strategy-proofness breaks down, and in conjunction with efficiency, we either end up with no rules or only with basically serial dictatorships. Of course, in serial dictatorships individuals who choose first have an advantage over individuals who choose later and these mechanisms treat individuals unequally. Another consequence of these results is that in the housing market with indifferences, individual rationality and efficiency are incompatible with either (strategy-proofness and nonbossiness) or group strategy-proofness.

In the housing market with strict preferences, only very few characterizations were obtained. An important paper by Ma (1994) showed that the top trading cycle algorithm is the only mechanism satisfying individual rationality, strategy-proofness ${ }^{5}$, and efficiency. Miyagawa (2002) showed that any mechanism satisfying individual rationality, strategy-proofness, non-bossiness, and anonymity must be either the top trading cycle algorithm or the no-trade mechanism. However, so far no characterization has been obtained in housing markets with indifferences. This is the purpose of our study.

Two recent and important contributions by Alcalde-Unzu and Molis (2011) and Jaramillo and Manjunath (2012) propose two distinct families of rules satisfying individual rationality, strategyproofness, and efficiency when indifferences are allowed. However, a foundation of the set of rules satisfying individual rationality, strategy-proofness, and efficiency is missing. In addition, as mentioned above, any rule satisfying these properties must be vulnerable to collusion because they all violate non-bossiness ${ }^{6}$. Furthermore, these two families are computationally complex for calculating the outcome. ${ }^{7}$

All characterizations in house allocation with or without indifferences share the following common feature: the characterized rules satisfy strategy-proofness and non-bossiness. Thus, we take as a basic set of axioms individual rationality, strategy-proofness, and non-bossiness. Now of course it is impossible for any such rule to be efficient and we can at most require weak efficiency: there is no allocation which all agents strictly prefer to the chosen allocation. In addition, we require consistency: if some group of agents are assigned only objects in this group, then we may remove those agents with their allotments (or endowments) without changing the allocation of the other

\footnotetext{
${ }^{4}$ Svensson (1994) was the first one to define serial dictatorships for the weak domain.

${ }^{5}$ Roth (1982) was the first to show that for strict preferences the top trading cycle algorithm satisfies strategyproofness.

${ }^{6}$ See also Proposition 2 in Jaramillo and Manjunath (2012).

${ }^{7}$ In Alcalde-Unzu and Molis (2011) it is not clear whether their algorithm is NP-hard or not whereas the algorithm by Jaramillo and Manjunath (2012) runs in polynomial $O\left(|N|^{5}\right)$ time (which tends to infinity very quickly).
} 
agents. This property is important in order to avoid appeals and the reallocation of the objects in some group of agents does not depend on the allotments of the other agents.

Using these properties we characterize a set of rules which is frequently used in real life: given a profile of weak preferences we break ties using some exogenously fixed tie-breakers. Then for the obtained strict profile we simply apply Gale's top trading cycle algorithm. We show that top trading cycle rules with fixed tie-breakers are the only rules satisfying individual rationality, strategyproofness, non-bossiness, weak efficiency, and consistency. A byproduct of our result is that any such mechanism chooses for each profile a competitive allocation. This reinforces the market-based approach even in the presence of indifferences. Related characterizations of competitive rules with fixed prices have been obtained by Barberà and Jackson (1995) and Miyagawa (2001) by using properties similar to ours. Furthermore, the proof reveals that our result is true for any domain in between the strict preferences and the weak preferences. Even if some indifferences are not allowed or seem implausible, our characterization of top trading with fixed tie-breaking remains true in those environments.

Our result supports a frequent practice in applications: exogenous fixed tie-breaking is applied to the weak preferences and then "good" rules from the strict domain are used. Top trading with fixed tie-breaking is simple and market designers may directly apply or implement it. Another recent example of such practice is Abdulkadiroğlu, Pathak and Roth (2009): in school choice with indifferences in priorities, equal priorities are broken according to fixed tie-breakers and then the standard student-proposing deferred-acceptance algorithm is applied to the profile of reported strict preferences and the obtained strict priorities. They defend such practice by the fact that no other strategy-proof mechanism Pareto dominates the DA-algorithm with fixed tie-breaking.

Variants of the top trading cycle algorithm have been proposed in a number of applications, for example for school choice by Abdulkadiroğlu and Sönmez (2003) and Kesten (2007, 2010) and in house allocation with existing tenants by Abdulkadiroğlu and Sönmez (1999). This algorithm plays also the important role in Pápai (2000), Larsson and Svensson (2005), and Ünver and Pycia (2009). In all these papers preferences are strict. Our result suggests that when preferences are weak, we break ties in a fixed manner and apply the corresponding variants of the top trading cycle algorithm to the obtained strict profile.

The paper is organized as follows. Section 2 introduces markets with indivisible goods. Section 3 presents the main result. Section 4 contains the proof of the main result. Section 5 discusses our main result.

\section{House Exchange}

Let $\mathcal{N}$ denote the finite set of potential agents. Agent $i$ 's object (or endowment or house) is denoted by $i$. Given $N \subseteq \mathcal{N}$ and $i \in N$, agent $i$ is equipped with a (weak) preference relation $R_{i}$ on $N$. Any such relation is reflexive, complete and transitive (but not necessarily strict). Let $P_{i}$ 
denote the strict relation associated with $R_{i}$ and $I_{i}$ the indifference relation associated with $R_{i}$. We assume that agent $i$ is never indifferent between his endowment $i$ and any other object, i.e. for all $j \in N \backslash\{i\}$, we have $j P_{i} i$ or $i P_{i} j$. This means agent $i$ is able to distinguish his endowment from any other object. Under this restriction any agent strictly prefers to keep his own object to any object with the same characteristics (because $i$ likes to avoid moving). In Section 5 we describe how to adjust our main result when indifferences with the endowment are allowed.

Let $\mathcal{R}_{i}^{N}$ denote the set of all such preference relations of agent $i$ on $N$. A relation $R_{i} \in \mathcal{R}_{i}^{N}$ is strict when for all $j, k \in N$ such that $j \neq k$, we have either $j P_{i} k$ or $k P_{i} j$. Let $\mathcal{P}_{i}^{N}$ denote the set of all strict preference relations of agent $i$ on $N$. Let $\mathcal{R}^{N}=\times_{i \in N} \mathcal{R}_{i}^{N}$ denote the set of all weak preference profiles and let $\mathcal{P}^{N}=\times_{i \in N} \mathcal{P}_{i}^{N}$ denote the set of all strict preference profiles. Given $R \in \mathcal{R}^{N}$ and $S \subseteq N$, let $R_{S}=\left(R_{i}\right)_{i \in S}$ denote the restriction of $R$ to $S$ and $R_{-S}=R_{N \backslash S}$. For any $i \in N$, let $\left.R_{i}\right|_{S}$ denote the restriction of $R_{i}$ to $S$, and let $\left.R\right|_{S}=\left(\left.R_{i}\right|_{S}\right)_{i \in N}$ denote the profile where each agent $i$ 's preference relation is restricted to $S$.

An economy consists of a finite set $N \subseteq \mathcal{N}$ and a profile $R \in \mathcal{R}^{N}$. Since agent $i$ 's endowment is denoted by $i$, an economy is for short a profile $R \in \mathcal{R}^{N}$. An allocation for $N$ is a mapping $\mu: N \rightarrow N$ such that for all $i, j \in N$ with $i \neq j, \mu(i) \neq \mu(j)$. Under any allocation each agent is receiving some object and no two agents receive the same object. Here $\mu(i)$ denotes the object received by agent $i$. Let $\mathcal{A}_{N}$ denote the set of all allocations for $N$. A(n allocation) rule is a mapping

$$
\varphi: \cup_{N \subseteq \mathcal{N}} \mathcal{R}^{N} \longrightarrow \cup_{N \subseteq \mathcal{N}} \mathcal{A}_{N}
$$

such that for all $N \subseteq \mathcal{N}$ and all $R \in \mathcal{R}^{N}$ we have $\varphi(R) \in \mathcal{A}_{N}$. Let $\varphi_{i}(R)$ denote the object assigned to $i$ by $\varphi$ for $R$.

Given $R \in \mathcal{R}^{N}$ and two allocations $\mu, \mu^{\prime} \in \mathcal{A}_{N}$, we write $\mu \sim^{R} \mu^{\prime}$ if for all $i \in N, \mu(i) I_{i} \mu^{\prime}(i)$. In other words, all agents view $\mu$ and $\mu^{\prime}$ welfare equivalent. Given two rules $\varphi$ and $\hat{\varphi}$, we write $\varphi \sim \hat{\varphi}$ if for all $N \subseteq \mathcal{N}$ and all $R \in \mathcal{R}^{N}$ we have $\varphi(R) \sim^{R} \hat{\varphi}(R)$. Then the rules $\varphi$ and $\hat{\varphi}$ are equivalent.

We will be interested in the following axioms.

Individual rationality means that each individual should always weakly prefer the assigned object to his endowment. If a rule is not individually rational, then agents are not necessarily willing to reallocate their endowments.

Individual Rationality: For all $N \subseteq \mathcal{N}$ and all $R \in \mathcal{R}^{N}$, we have $\varphi_{i}(R) R_{i} i$ for all $i \in N$.

Strategy-proofness means that no individual can manipulate the mechanism to his advantage by misreporting his preference. This incentive-compatibility condition ensures that agents report truthfully and the allocation chosen by the rule is based on true preferences.

Strategy-Proofness: For all $N \subseteq \mathcal{N}$, all $R \in \mathcal{R}^{N}$, all $i \in N$, and all $R_{i}^{\prime} \in \mathcal{R}_{i}^{N}$, we have 
$\varphi_{i}(R) R_{i} \varphi_{i}\left(R_{i}^{\prime}, R_{-i}\right)$

Weak efficiency means that it is impossible to make all agents strictly better off through some other allocation. Note that this requirement is very weak.

Weak Efficiency: For all $N \subseteq \mathcal{N}$ and all $R \in \mathcal{R}^{N}$, there exists no $\mu \in \mathcal{A}_{N}$ such that $\mu(i) P_{i} \varphi_{i}(R)$ for all $i \in N$.

Non-bossiness means that no individual can change the allocation without changing his assigned object. This property prevents collusion among agents because if a mechanism violates this condition, then an agent may be bribed by others in order to change the allocation without changing his assigned object.

Non-Bossiness: For all $N \subseteq \mathcal{N}$, all $R \in \mathcal{R}^{N}$, all $i \in N$, and all $R_{i}^{\prime} \in \mathcal{R}_{i}^{N}$, if $\varphi_{i}(R)=\varphi_{i}\left(R_{i}^{\prime}, R_{-i}\right)$, then $\varphi(R)=\varphi\left(R_{i}^{\prime}, R_{-i}\right){ }^{8}$

Consistency requires that whenever for a profile some set of agents reallocates their endowments, then we may remove these agents with their endowments from the economy without changing the allocation chosen for the other agents. This property implies that the mechanism is immune with respect to appeals where some set of agents may ask to reallocate their endowments.

Consistency: For all $S \subseteq N \subseteq \mathcal{N}$ and all $R \in \mathcal{R}^{N}$, if $\cup_{i \in N \backslash S}\left\{\varphi_{i}(R)\right\}=N \backslash S$, then $\varphi_{i}\left(\left.R_{S}\right|_{S}\right)=\varphi_{i}(R)$ for all $i \in S .{ }^{910}$

Note that this condition is much weaker than the property used in economies without individual endowments where any set of agents can leave with their allotments.

\section{Top trading with fixed tie-breaking}

Given $R_{i} \in \mathcal{R}_{i}^{N}$ and $R_{i}^{\prime} \in \mathcal{P}_{i}^{N}$, we say $R_{i}^{\prime}$ is a strict transformation of $R_{i}$ if for all $j, k \in N$, we have $j P_{i} k \Rightarrow j P_{i}^{\prime} k$. Given $R \in \mathcal{R}^{N}$, we say that $R^{\prime} \in \mathcal{P}^{N}$ is a strict transformation of $R$ if for all $i \in N$, $R_{i}^{\prime}$ is a strict transformation of $R_{i}$. Let $S T(R)$ denote the set of all strict transformations of $R$. Of

\footnotetext{
${ }^{8}$ Note that under this condition, if an agent changes his report from $R_{i}$ to $R_{i}^{\prime}$ and is assigned the same object, then independently of his true preference relation agent $i$ is indifferent between the two reports $R_{i}$ and $R_{i}^{\prime}$ (given $\left.R_{-i}\right)$ and all other agents are indifferent independently of their true preferences if the same allocation is chosen under $R$ and $\left(R_{i}^{\prime}, R_{-i}\right)$.

${ }^{9}$ Note that the part $\cup_{i \in N \backslash S}\left\{\varphi_{i}(R)\right\}=N \backslash S$ may equivalently be replaced by $\cup_{i \in S}\left\{\varphi_{i}(R)\right\}=S$.

${ }^{10}$ Thomson (1992) proposes to use the name "separation independence". For a survey of consistent allocation rules, see Thomson (2009).
} 
course, for strict $R$ we have $S T(R)=\{R\}$.

Note that in $S T(R)$ ties can be broken arbitrarily. We will be interested in the case where ties are broken in a fixed manner. Let $\succ_{i} \in \mathcal{P}_{i}^{\mathcal{N}}$ denote a fixed tie-breaker. This tie-breaker will be used to break ties in any weak preference relation of $i$ in order to obtain a strict preference relation. Given $R_{i} \in \mathcal{R}_{i}^{N}$, let $\succ_{i}\left(R_{i}\right)$ denote the strict transformation $R_{i}^{\prime} \in \mathcal{P}_{i}^{N}$ of $R_{i}$ such that for all $j, k \in N$ with $j \neq k$, if $j I_{i} k$ and $j \succ_{i} k$, then $j P_{i}^{\prime} k$.

Let $\succ=\left(\succ_{i}\right)_{i \in \mathcal{N}}$. For any $R \in \mathcal{R}^{N}$, let $\succ(R)=\left(\succ_{i}\left(R_{i}\right)\right)_{i \in N}$. Obviously, if $R \in \mathcal{P}^{N}$, then $\succ(R)=R$. For any strict $R$, let $f(R)$ denote the outcome of Gale's top trading cycles algorithm. For a formal description of the algorithm we refer the reader to Pápai (2000). Informally, the algorithm works as follows: for any $N$ and any profile $R \in \mathcal{P}^{N}$, each agent points to his most preferred object (or agent). Because $N$ is finite, there must be at least one (top) cycle and for each top cycle agents trade their endowments (as specified by the cycle). The objects of these trading cycles are deleted from the preferences of the remaining agents and we apply the same procedure to the remaining agents and their preferences restricted to the remaining objects (or agents), and so on.

Given a profile of fixed tie-breakers $\succ$, let $f^{\succ}$ denote Gale's top trading cycles algorithm with fixed tie-breaking $\succ$ : for all $R \in \mathcal{R}^{N}$ we have

$$
f^{\succ}(R)=f(\succ(R))
$$

In other words, in any weak preference profile ties are broken according to $\succ$ and then Gale's top trading cycles algorithm is applied to the resulting strict profile.

The following is our main result.

Theorem 1. (a) For any profile of fixed tie-breakers $\succ, f^{\succ}$ satisfies individual rationality, strategy-proofness, weak efficiency, non-bossiness, and consistency.

(b) If a rule $\varphi$ satisfies individual rationality, strategy-proofness, weak efficiency, non-bossiness, and consistency, then there exists a profile $\succ$ of fixed tie-breakers such that $\varphi \sim f^{\succ}$.

Theorem 1 gives a justification for the following common practice in real life: indifferences are resolved using some fixed tie-breaking and the "good and simple" top trading cycle algorithm is applied to the resulting profile of strict preferences. Of course, (b) of Theorem 1 says that any rule satisfying our properties must be equivalent to a top trading cycle rule with fixed tie-breaking. It must not necessarily coincide with such a rule because sometimes there are "many" indifferences and another utility-equivalent allocation may be chosen without violating our conditions. In a sense, this is not surprising because agents only care about their utilities and not about the exact allocation. The following example gives a rule which satisfies all our properties but which does not coincide with a top trading cycle rule with fixed tie-breaking. 
Example 1. Let $\mathcal{N}=\{1,2,3,4\}$ and $\succ=\left(\succ_{1}, \succ_{2}, \succ_{3}, \succ_{4}\right)$ be given by:

\begin{tabular}{c|c|c|c}
$\succ_{1}$ & $\succ_{2}$ & $\succ_{3}$ & $\succ_{4}$ \\
\hline 2 & 3 & 4 & 1 \\
3 & 4 & 1 & 2 \\
4 & 2 & 2 & 3 \\
1 & 1 & 3 & 4
\end{tabular}.

We define the rule $\phi$ : for all $N \subseteq \mathcal{N}$ and all $R \in \mathcal{R}^{N}$, (i) if $N=\mathcal{N}, f^{\succ}(R)=(2,3,4,1)$, and both $4 \in \operatorname{top}\left(R_{1}\right)$ and $2 \in \operatorname{top}\left(R_{3}\right)$ (where $\operatorname{top}\left(R_{i}\right)$ denotes the set of most $R_{i}$-preferred elements), then $\phi(R)=(4,3,2,1)$; and (ii) otherwise $\phi(R)=f^{\succ}(R)$.

Note that $\phi \sim f^{\succ}$ and $\phi \neq f^{\succ}$ because for profiles $R$ of type (i), we have $\phi(R)=(4,3,2,1)$ whereas $f^{\succ}(R)=(2,3,4,1)$. Since $\phi \sim f^{\succ}$, from (a) of Theorem 1 it is obvious that $\phi$ satisfies individual rationality, strategy-proofness, and weak efficiency.

In order to check that $\phi$ satisfies non-bossiness, let $R \in \mathcal{R}^{N}, i \in N$, and $R_{i}^{\prime} \in \mathcal{R}_{i}^{N}$ be such that $\phi_{i}(R)=\phi_{i}\left(R_{i}^{\prime}, R_{-i}\right)$. Now if both $R$ and $\left(R_{i}^{\prime}, R_{-i}\right)$ are profiles of type (i) or both $R$ and $\left(R_{i}^{\prime}, R_{-i}\right)$ are profiles of type (ii), then non-bossiness is satisfied. Suppose that $R$ and $\left(R_{i}^{\prime}, R_{-i}\right)$ are not profiles of the same type. Then $N=\mathcal{N}$ and say $R$ is a profile of type (i) and $\left(R_{i}^{\prime}, R_{-i}\right)$ is a profile of type (ii).

First, suppose that $i \in\{2,4\}$. Then $\phi_{i}(R)=f_{i}^{\succ}(R)$. We also have $\phi_{i}\left(R_{i}^{\prime}, R_{-i}\right)=f_{i}^{\succ}\left(R_{i}^{\prime}, R_{-i}\right)$ because $\left(R_{i}^{\prime}, R_{-i}\right)$ is of type (ii). By $\phi_{i}(R)=\phi_{i}\left(R_{i}^{\prime}, R_{-i}\right)$, we now have $f_{i}^{\succ}(R)=f_{i}^{\succ}\left(R_{i}^{\prime}, R_{-i}\right)$. Thus, by non-bossiness of $f^{\succ}, f^{\succ}(R)=f^{\succ}\left(R_{i}^{\prime}, R_{-i}\right)=(2,3,4,1)$. Since $i \in\{2,4\}$, we now again have $4 \in \operatorname{top}\left(R_{1}\right)$ and $2 \in \operatorname{top}\left(R_{3}\right)$ which means that $\left(R_{i}^{\prime}, R_{-i}\right)$ is of type (i), a contradiction.

Second, suppose that $i \in\{1,3\}$, say $i=1$ (the case $i=3$ is similar). If $2 \in \operatorname{top}\left(R_{1}^{\prime}\right)$, then $f_{1}^{\succ}\left(R_{1}^{\prime}, R_{-1}\right)=2 \neq 4=\phi_{1}(R)$. Since $\left(R_{1}^{\prime}, R_{-1}\right)$ is of type (ii), we have $\phi_{1}\left(R_{1}^{\prime}, R_{-1}\right)=2$ which contradicts the fact $\phi_{1}\left(R_{1}^{\prime}, R_{-1}\right)=\phi_{1}(R)$. Thus, $2 \notin \operatorname{top}\left(R_{1}^{\prime}\right)$. If $3 \in \operatorname{top}\left(R_{1}^{\prime}\right)$, then $f^{\succ}\left(R_{1}^{\prime}, R_{-1}\right)=$ $(3,2,4,1)$. Then $\phi_{1}\left(R_{1}^{\prime}, R_{-1}\right)=3 \neq 4$ because $\left(R_{1}^{\prime}, R_{-1}\right)$ is of type (ii). This contradicts the fact $\phi_{1}\left(R_{1}^{\prime}, R_{-1}\right)=\phi_{1}(R)$. Thus, $\operatorname{top}\left(R_{1}^{\prime}\right)=\{4\}$. But then $f^{\succ}\left(R_{1}^{\prime}, R_{-1}\right)=(4,3,2,1)$. Because $\left(R_{1}^{\prime}, R_{-1}\right)$ is of type (ii), we now have $\phi\left(R_{1}^{\prime}, R_{-1}\right)=f^{\succ}\left(R_{1}^{\prime}, R_{-1}\right)$. But now $\phi\left(R_{1}^{\prime}, R_{-1}\right)=\phi(R)$, and $\phi$ satisfies non-bossiness.

In order to check that $\phi$ satisfies consistency, let $R \in \mathcal{R}^{N}$. If $R$ is of type (ii), then this is obvious. Let $R$ be of type (i). But then we may remove only $\{1,4\}$ or $\{2,3\}$ from $R$ and consistency is satisfied.

Therefore, $\phi$ satisfies all the properties in Theorem 1 but $\phi \neq f^{\succ}$. Of course, there exists no other profile of tie-breakers $\succ^{\prime}$ such that $\phi=f^{\succ^{\prime}}$ because $\phi$ is welfare equivalent to $f^{\succ}$ and for $\succ \neq \succ^{\prime}$ we have $f^{\succ} \not f^{\succ^{\prime}}$.

Remark 1. Regarding the proof of Theorem 1, (a) follows straightforwardly from the fact that the top trading cycles algorithm satisfies all the properties on the strict domain and ties are broken 
exogenously. In proving (b), we first show that any rule satisfying our properties must coincide with the top trading cycles algorithm for strict preferences. Using this fact, then we show that for any preference profile, the rule must choose the outcome of the top trading cycles algorithm for some strict transformation of the profile. Then we construct for each agent $i$ a tie-breaker $\succ_{i}$ and show that for any profile and any top cycle of the top trading cycles algorithm with these tie-breakers, all agents in this trading cycle must receive one of their most preferred objects. Then we show that for any profile and any top cycle of the top trading cycles algorithm with these tie-breakers, each agent in this trading cycle must receive an endowment of one of the agents belonging to the trading cycle. This and the previous step are non-straightforward because of handling agents with indifferences. Finally, using these two previous steps, then we can show (b) with consistency.

We relate Theorem 1 to the core and to the set of competitive allocations in a housing market. Given $R \in \mathcal{R}^{N}, T \subseteq N$, and $\mu \in \mathcal{A}_{N}$, if for some $\bar{\mu} \in \mathcal{A}_{T}$, (i) for all $i \in T, \bar{\mu}(i) R_{i} \mu(i)$, and (ii) for some $j \in T, \bar{\mu}(j) P_{j} \mu(j)$, then we say that coalition $T$ blocks $\mu$ under $R$. The core chooses for each profile the allocations that are not blocked by any coalition.

Given a preference profile $R \in \mathcal{R}^{N}$, an allocation is called competitive when there exist prices for all $i \in N$ such that each agent maximizes his preference on the set of affordable objects and the price of his assignment at $\mu$ equals the price of his initial endowment. We consider the solution that chooses for each preference profile its set of competitive allocations, called the competitive solution. Formally, given $R \in \mathcal{R}^{N}, \mu \in \mathcal{A}_{N}$ is a competitive equilibrium if there exists a price vector $(p(1), p(2), \ldots, p(n)) \in \mathbb{R}^{N}$ such that for all $i \in N$, (i) $p(\mu(i))=p(i)$; and (ii) for all $j \in N$, if $p(j) \leq p(i)$, then $\mu(i) R_{i} j$. Now a rule is competitive if it chooses for any profile a competitive allocation.

When preferences are linear orders, the core contains exactly one allocation, which is the unique competitive equilibrium (Roth and Postlewaite, 1977). When preferences are weak orders, the core is always a subset of the competitive solution (Wako, 1984). Moreover, for each profile, the set of competitive allocations is obtained by computing the cores of all its strict transformations (Shapley and Scarf, 1974). ${ }^{11}$ Now of course, any top trading cycles algorithm with fixed tie-breaking chooses for each profile a competitive allocation. This supports the market-based approach even when preferences are weak, also because competitive allocations always exist whereas the core may be empty.

Top trading with fixed tie-breaking can be seen as competitive rules with fixed tie-breaking. Of course, those rules are not efficient ${ }^{12}$ (and therefore, the rules proposed by Alcalde-Unzu and Molis (2011) and Jaramillo and Manjunath (2012) are not competitive) as the following example shows:

\footnotetext{
${ }^{11}$ Ehlers (2004) shows that the minimal monotonic extension of the core is the correspondence choosing for each profile its set of competitive allocations. Recently, Klaus, Klijn and Walzl (2010) established on the domain $\mathcal{R}^{N}$ that the set of competitive allocations is the unique von Neumann-Morgenstern farsightedly stable set based on weak dominance.

${ }^{12}$ Efficiency: For all $N \subseteq \mathcal{N}$ and all $R \in \mathcal{R}^{N}$, there exists no $\mu \in \mathcal{A}_{N}$ such that $\mu(i) R_{i} \varphi_{i}(R)$ for all $i \in N$, and $\mu(j) P_{j} \varphi_{j}(R)$ for some $j \in N$.
} 
let $N=\{1,2,3\}$ and consider $R \in \mathcal{R}^{N}$

\begin{tabular}{c|c|c}
$R_{1}$ & $R_{2}$ & $R_{3}$ \\
\hline 2,3 & 1 & 1 \\
1 & 3 & 2 \\
& 2 & 3
\end{tabular}.

Now $(2,1,3)$ and $(3,2,1)$ are the competitive allocations under $R$ whereas $(3,1,2)$ and $(2,3,1)$ are the efficient allocations under $R$. Hence, there is no competitive rule is efficient in this example (and no efficient rule is competitive). In a sense this is not surprising because our rules are competitive with fixed tie-breaking. The same feature is shared in Barberà and Jackson (1995) where in classical exchange economies competitive rules with fixed prices (or fixed proportions) are characterized and in Miyagawa (2001) where with indivisible goods and monetary compensations competitive rules with fixed prices are characterized. Interestingly, individual rationality, strategy-proofness, and non-bossiness are common properties used in Theorem 1 and their characterizations. Note, however, in our main result tie-breaking is fixed but not prices.

Remark 2 (Group Manipulations). Group strategy-proofness means that no group of agents is able to manipulate the mechanism such that all members of the group become weakly better off and some are strictly better off. Weak group strategy-proofness means that no group of agents is able to manipulate the mechanism such that all members of the group become strictly better off.

Group Strategy-Proofness: For all $N \subseteq \mathcal{N}$, all $R \in \mathcal{R}^{N}$, and all $S \subseteq N$, there exists no $R_{S}^{\prime} \in \times_{i \in S} \mathcal{R}_{i}^{N}$ such that $\varphi_{i}\left(R_{S}^{\prime}, R_{-S}\right) R_{i} \varphi_{i}(R)$ for all $i \in S$ with strict preference holding for some $j \in S$.

Weak Group Strategy-Proofness: For all $N \subseteq \mathcal{N}$, all $R \in \mathcal{R}^{N}$, and all $S \subseteq N$, there exists no $R_{S}^{\prime} \in \times_{i \in S} \mathcal{R}_{i}^{N}$ such that $\varphi_{i}\left(R_{S}^{\prime}, R_{-S}\right) P_{i} \varphi_{i}(R)$ for all $i \in S$.

Pápai (2000) showed that under strict preferences, strategy-proofness and non-bossiness are equivalent to group strategy-proofness whereas from Ehlers (2002) it follows that group strategyproofness and efficiency are incompatible on the domain $\mathcal{R}^{N}$ when $|N| \geq 3$. Furthermore, top trading with fixed tie-breaking is not group strategy-proof ${ }^{13}$ but is weakly group strategy-proof (Bird, 1984). ${ }^{14}$ Therefore, in Theorem 1 strategy-proofness may be replaced by the stronger (or more robust) non-manipulability notion of weak group strategy-proofness without altering its conclusions.

\footnotetext{
${ }^{13}$ In the above example, if $2 \succ_{1} 3$, then choose $R_{1}^{\prime}$ such that $3 P_{1}^{\prime} 2 P_{1}^{\prime} 1, S=\{1,3\}$, and $R_{3}^{\prime}=R_{3}$; and if $3 \succ_{1} 2$, then choose $R_{1}^{\prime}$ such that $2 P_{1}^{\prime} 3 P_{1}^{\prime} 1, S=\{1,2\}$ and $R_{2}^{\prime}=R_{2}$.

${ }^{14}$ It is not known whether the rules proposed by Alcalde-Unzu and Molis (2011) and Jaramillo and Manjunath (2012) satisfy weak group strategy-proofness (or even whether individual rationality, efficiency and weak group strategy-proofness are compatible).
} 


\section{Proof of Theorem 1}

We show (a) of Theorem 1. Let $\succ$ be a profile of tie-breakers.

First, $f^{\succ}$ satisfies individual rationality: let $R \in \mathcal{R}^{N}$ and $i \in N$; since $f$ satisfies individual rationality on the strict domain $\mathcal{P}^{N}$, we have $f_{i}^{\succ}(R) \succ_{i}\left(R_{i}\right) i$. Since $\succ_{i}$ breaks the ties in $R_{i}$, we now also have $f_{i}^{\succ}(R) R_{i} i$, the desired conclusion.

Second, $f^{\succ}$ satisfies strategy-proofness: let $R \in \mathcal{R}^{N}, i \in N$, and $R_{i}^{\prime} \in \mathcal{R}_{i}^{N}$; since $f$ satisfies strategy-proofness on the strict domain $\mathcal{P}^{N}$, we have $f_{i}^{\succ}(R) \succ_{i}\left(R_{i}\right) f_{i}^{\succ}\left(R_{i}^{\prime}, R_{-i}\right)$. Since $\succ_{i}$ breaks the ties in $R_{i}$, we now also have $f_{i}^{\succ}(R) R_{i} f_{i}^{\succ}\left(R_{i}^{\prime}, R_{-i}\right)$, the desired conclusion.

Third, $f^{\succ}$ satisfies weak efficiency: let $R \in \mathcal{R}^{N}$ and $\mu \in \mathcal{A}_{N}$; if $\mu(i) P_{i} f_{i}^{\succ}(R)$ for all $i \in N$, then $\mu(i) \succ_{i}\left(R_{i}\right) f_{i}^{\succ}(R)$ for all $i \in N$ (with strict preference holding for all $i \in N$ ). Obviously this contradicts the fact that $f$ satisfies weak efficiency on the strict domain $\mathcal{P}^{N}$.

Fourth, $f^{\succ}$ satisfies non-bossiness: let $R \in \mathcal{R}^{N}, i \in N$, and $R_{i}^{\prime} \in \mathcal{R}_{i}^{N}$ be such that $f_{i}^{\succ}(R)=$ $f_{i}^{\succ}\left(R_{i}^{\prime}, R_{-i}\right)$; since $f$ satisfies non-bossiness on the strict domain $\mathcal{P}^{N}$, we have $f_{i}(\succ(R))=f_{i}(\succ$ $\left.\left(R_{i}^{\prime}, R_{-i}\right)\right)$ implies $f(\succ(R))=f\left(\succ\left(R_{i}^{\prime}, R_{-i}\right)\right)$. Thus, $f^{\succ}(R)=f^{\succ}\left(R_{i}^{\prime}, R_{-i}\right)$.

Fifth, $f^{\succ}$ satisfies consistency: let $R \in \mathcal{R}^{N}$ and $S \subseteq N$ be such that $\cup_{i \in N \backslash S}\left\{f_{i}^{\succ}(R)\right\}=N \backslash S$; since $f$ satisfies consistency on the strict domain $\mathcal{P}^{N}$ we have $f_{i}\left(\succ\left(\left.R_{-S}\right|_{N \backslash S}\right)\right)=f_{i}(\succ(R))$ for all $i \in N \backslash S$. Thus, $f_{i}^{\succ}\left(\left.R_{-S}\right|_{N \backslash S}\right)=f_{i}^{\succ}(R)$ for all $i \in N \backslash S$.

We prove (b) of Theorem 1 in a number of lemmas. Let $\varphi$ a be rule satisfying individual rationality, strategy-proofness, weak efficiency, non-bossiness, and consistency. The roadmap of our proof is described in Remark 1.

We will often use the following convention: given $N \subseteq \mathcal{N}$ and distinct $i, j, k, l \in N, R_{i}: j, k, l, \ldots$ means we choose some $R_{i} \in \mathcal{P}_{i}^{N}$ such that $j P_{i} k P_{i} l$ and $l P_{i} h$ for all $h \in N \backslash\{j, k, l\}$. Throughout, let $N \subseteq \mathcal{N}$.

For a given (trading) coalition $S \subseteq N$, a (trading) cycle is an allocation $\mu \in \mathcal{A}_{S}$ such that there exists a relabeling $\left(i_{1}, \ldots, i_{|S|}\right)$ of the agents in $S$ such that $\mu\left(i_{l}\right)=i_{l+1}$ for $l \in\{1, \ldots,|S|-1\}$ and $\mu\left(i_{|S|}\right)=i_{1}$. We then sometimes write for this cycle $c=\left(i_{2}, \ldots, i_{|S|}, i_{1}\right)$ and call $S$ the trading coalition of $c$.

Obviously, any $\mu \in \mathcal{A}_{N}$ partitions $N$ into cycles.

Lemma 1. For all $R \in \mathcal{P}^{N}$, we have $\varphi(R)=f(R)$.

Proof. Let $R \in \mathcal{P}^{N}$ and let $c$ be a top cycle in $f(R)$ with trading coalition $C$. Without loss of generality, let $C=\{1, \ldots, k\}$ and $c=(2,3, \ldots, k, 1)$ (where 1 receives 2,2 receives $3, \ldots, k$ receives 1). We show that $\varphi_{i}(R)=f_{i}(R)$ for all $i \in\{1, \ldots, k\}$. If $k=1$, then by individual rationality, $\varphi_{1}(R)=1=f_{1}(R)$, the desired conclusion. 
Let $k \geq 2$ and suppose that $\varphi_{k}(R) \neq 1$. Let $R_{k}^{\prime}: 1, k, \ldots$ By strategy-proofness and $\varphi_{k}(R) \neq 1$, we have $\varphi_{k}\left(R_{k}^{\prime}, R_{-k}\right) \neq 1$. By individual rationality, $\varphi_{k}\left(R_{k}^{\prime}, R_{-k}\right)=k$. Thus, $\varphi_{k-1}\left(R_{k}^{\prime}, R_{-k}\right) \neq k$. Note that $c$ is still a top cycle under $\left(R_{k}^{\prime}, R_{-k}\right)$ and $f_{k-1}\left(R_{k}^{\prime}, R_{-k}\right)=k$. Similarly as above we may replace $R_{k-1}$ with $R_{k-1}^{\prime}: k, k-1, \ldots$ and obtain the same conclusions.

Finally, we obtain the profile $\left(R_{\{1, \ldots, k\}}^{\prime}, R_{-\{1, \ldots, k\}}\right)$ where $\varphi_{i}\left(R_{\{1, \ldots, k\}}^{\prime}, R_{-\{1, \ldots, k\}}\right)=i$ for all $i \in\{1, \ldots, k\}$. Now by consistency, $\varphi_{i}\left(\left.R_{\{1, \ldots, k\}}^{\prime}\right|_{\{1, \ldots, k\}}\right)=i$ for all $i \in\{1, \ldots, k\}$, which is a contradiction to weak efficiency becaus $c \in A_{\{1, \ldots, k\}}$ and $c(i) P_{i} i$ for all $i \in\{1, \ldots, k\}$.

Hence, we must have $\varphi_{i}(R)=f_{i}(R)$ for all $i \in\{1, \ldots, k\}$. Now by consistency, we have for all $i \in N \backslash\{1, \ldots, k\}$ both $\varphi_{i}\left(\left.R_{-\{1, \ldots, k\}}\right|_{N \backslash\{1, \ldots, k\}}\right)=\varphi_{i}(R)$ and $f_{i}\left(\left.R_{-\{1, \ldots, k\}}\right|_{N \backslash\{1, \ldots, k\}}\right)=f_{i}(R)$. Now by the same arguments as above any top cycle must be executed for $\left.R_{-\{1, \ldots, k\}}\right|_{N \backslash\{1, \ldots, k\}}$ by both rules $\varphi$ and $f$.

Because $N$ is finite, we must have $\varphi(R)=f(R)$, the desired conclusion.

Remark 3. Note that the proof of Lemma 1 establishes a new characterization of Gale's top trading cycles algorithm on the domain of strict preferences: $f$ is the only rule satisfying individual rationality, strategy-proofness, weak efficiency, and consistency. Note that in this characterization consistency is independent from the other properties: let $e \in \mathcal{A}_{\mathcal{N}}$ be such that $e(i)=i$ for all $i \in \mathcal{N}$; now for all $N \subseteq \mathcal{N}$ and all $R \in \mathcal{P}^{N}$, (i) if for some $i \in N, R_{i}: i, \ldots$, then $\phi(R)=e_{N}$ (where $e_{N}=(e(i))_{i \in N}$ ), and (ii) otherwise $\phi(R)=f(R)$. It is easy to check that $\phi$ satisfies individual rationality, strategy-proofness, and weak efficiency, and that $\phi$ violates consistency.

Lemma 2. For all $R \in \mathcal{R}^{N}$, there exists $R^{\prime} \in S T(R)$ such that $\varphi(R)=f\left(R^{\prime}\right)$.

Proof. Let $R^{\prime} \in S T(R)$ be such that for all $i, j \in N$, if $\varphi_{i}(R) \neq j$ and $\varphi_{i}(R) R_{i} j$, then $\varphi_{i}(R) P_{i}^{\prime} j$. It is easy to see from strategy-proofness and non-bossiness that we must have $\varphi(R)=\varphi\left(R^{\prime}\right)$. Now $R^{\prime} \in \mathcal{P}^{N}$ and by Lemma 1, $\varphi\left(R^{\prime}\right)=f\left(R^{\prime}\right)$. Hence, $\varphi(R)=f\left(R^{\prime}\right)$, the desired conclusion.

Next we define for any $i \in \mathcal{N}$ the tie-breaker $\succ_{i}$. For any $j, k \in \mathcal{N}$ such that $|\{i, j, k\}|=3$, consider the profile $R \in \mathcal{R}^{\{i, j, k\}}$ such that

\begin{tabular}{c|c|c}
$R_{i}$ & $R_{j}$ & $R_{k}$ \\
\hline$j, k$ & $i$ & $i$ \\
$i$ & $j$ & $k$ \\
& $k$ & $j$.
\end{tabular}

Now by Lemma 2 , we have $\left[\varphi_{i}(R)=j\right.$ and $\left.\varphi_{j}(R)=i\right]$ or $\left[\varphi_{i}(R)=k\right.$ and $\left.\varphi_{k}(R)=i\right]$. Now if $\varphi_{j}(R)=i$, then we set $j \succ_{i} k$, and if $\varphi_{k}(R)=i$, then we set $k \succ_{i} j$. Furthermore, we set both $j \succ_{i} i$ and $k \succ_{i} i$.

Obviously, $\succ_{i}$ is reflexive, complete, and strict. Now $\succ_{i}$ is a tie-breaker if $\succ_{i}$ is transitive.

Lemma 3. For all $i \in \mathcal{N}, \succ_{i}$ is transitive. 
Proof. Let $j \succ_{i} k$ and $k \succ_{i} l$. We need to show $j \succ_{i} l$.

If $i \in\{j, k, l\}$, then by construction we must have $l=i$. By definition, we then have $j \succ_{i} i$, the desired conclusion.

Let $i \notin\{j, k, l\}$. Consider the profile $R \in \mathcal{R}^{\{i, j, k, l\}}$ such that

\begin{tabular}{c|c|c|c}
$R_{i}$ & $R_{j}$ & $R_{k}$ & $R_{l}$ \\
\hline$j, k, l$ & $i$ & $i$ & $i$ \\
$i$ & $j$ & $k$ & $l$ \\
& $k$ & $j$ & $j$ \\
& $l$ & $l$ & $k$
\end{tabular}

Now by Lemma 2, we have $\varphi_{i}(R) \neq i$. Suppose that $\varphi_{i}(R) \neq j$. By individual rationality, $\varphi_{j}(R)=j$. Now by consistency and $k \succ_{i} l$, we must have $\varphi_{i}(R)=k$. Hence, $\varphi_{l}(R)=l$. Now again by consistency, $\varphi_{i}\left(\left.R_{-l}\right|_{\{i, j, k\}}\right)=k$ and $\varphi_{k}\left(\left.R_{-l}\right|_{\{i, j, k\}}\right)=i$. Note that $\left.R_{-l}\right|_{\{i, j, k\}}$ is identical to the profile in (1) which was used for the definition of $\succ_{i}$ on $\{j, k\}$. This is a contradiction to $j \succ_{i} k$.

By Lemma 3, $\succ=\left(\succ_{i}\right)_{i \in \mathcal{N}}$ is a well-defined profile of tie-breakers.

We will use the following convention: whenever we write $c$ is a top cycle in $f^{\succ}(R)$, then we mean that $c$ is a top cycle in $f(\succ(R))$.

The following is the first key to the proof of Theorem 1. In the proof of Lemma 4, we want to show that for all $R$, if $c$ is a top cycle in $f^{\succ}(R)$ with trading coalition $C$, then we have $\varphi_{i}(R) I_{i} f_{i}^{\succ}(R)$ for all $i \in C$. When this does not hold, the difficulty here and in all subsequent lemmas is to handle any agent $i$ where $\varphi_{i}(R) I_{i} f_{i}^{\succ}(R)$ and $\varphi_{i}(R) \neq f_{i}^{\succ}(R)$. This is because for any such agent we cannot change his preference relation to a strict relation without losing the above indifference because our axioms do not impose any requirement for such changes.

For any $R_{i} \in \mathcal{R}_{i}^{N}$, let $\operatorname{top}\left(R_{i}\right)$ denote the set of most preferred objects under $R_{i}$ in $N$.

Lemma 4. For all $R \in \mathcal{R}^{N}$, if c is a top cycle in $f^{\succ}(R)$ with trading coalition $C$, then $\varphi_{i}(R) I_{i} f_{i}^{\succ}(R)$ for all $i \in C$.

Proof. Let $R \in \mathcal{R}^{N}$ and let $c$ be a top cycle in $f^{\succ}(R)$ with trading coalition $C$. Without loss of generality, let $C=\{1, \ldots, k\}$ and $c=(2,3, \ldots, k, 1)$ (where 1 receives 2,2 receives $3, \ldots, k$ receives 1). Note that for all $i \in\{1, \ldots, k\}, f_{i}^{\succ}(R) R_{i} \varphi_{i}(R)$. We show that $\varphi_{i}(R) I_{i} f_{i}^{\succ}(R)$ for all $i \in\{1, \ldots, k\}$.

We show Lemma 4 first for cycles of length one and length two, and then by induction for cycles of length greater than or equal to three.

If $k=1$, then by individual rationality and the fact that there is no $i \neq 1$ such that $i I_{1} 1$, $\varphi_{1}(R)=1=f_{1}^{\succ}(R)$, the desired conclusion.

If $k=2$ and $f_{i}^{\succ}(R) P_{i} \varphi_{i}(R)$ for some $i \in\{1,2\}$, say $i=2$, then let $R_{2}^{\prime}: 1,2, \ldots$ By individual rationality and strategy-proofness, $\varphi_{2}\left(R_{2}^{\prime}, R_{-2}\right)=2$. Note that by consistency and individ- 
ual rationality, for $R_{1}^{\prime}: 2,1, \ldots$ we have $\varphi_{1}\left(R_{1}^{\prime}, R_{2}^{\prime}, R_{-1,2}\right)=1$. Thus, by strategy-proofness, $\varphi_{1}\left(R_{2}^{\prime}, R_{-2}\right) \in \operatorname{top}\left(R_{1}\right)$ and $\left|\operatorname{top}\left(R_{1}\right)\right| \geq 2$. Let $c^{\prime}$ denote the cycle along which 1 trades in $\varphi\left(R_{2}^{\prime}, R_{-2}\right)$, and let $C^{\prime}$ be this coalition. Now by consistency, we may suppose $N=C^{\prime} \cup\{2\}$. By strategy-proofness and non-bossiness, we may suppose that for all $i \in C^{\prime} \backslash\{1,2\}, R_{i}: \varphi_{i}(R), i, \ldots$. Let $\varphi_{1}\left(R_{2}^{\prime}, R_{-2}\right)=l$. Note that $l=1$ is impossible because $2 P_{1} 1$ from the fact that $(2,1)$ is a top cycle in $f^{\succ}\left(R_{2}^{\prime}, R_{-2}\right)$ and from strategy-proofness, $l \in \operatorname{top}\left(R_{1}\right)$. Let $\varphi_{l}\left(R_{2}^{\prime}, R_{-2}\right)=j$ and $R_{l}^{\prime}: j, 1, l, \ldots$ By strategy-proofness and non-bossiness, $\varphi\left(R_{2}^{\prime}, R_{l}^{\prime}, R_{-2, l}\right)=\varphi\left(R_{2}^{\prime}, R_{-2}\right)$. Let $R_{l}^{\prime \prime}: 1, j, l, \ldots$ Now by $\varphi_{l}\left(R_{2}^{\prime}, R_{l}^{\prime \prime}, R_{-2, l}\right)=j$, individual rationality and strategy-proofness, $\varphi_{l}\left(R_{2}^{\prime}, R_{l}^{\prime \prime}, R_{-2, l}\right) \in\{1, j\}$. If $\varphi_{l}\left(R_{2}^{\prime}, R_{l}^{\prime \prime}, R_{-2, l}\right)=j$, then by non-bossiness, $\varphi\left(R_{2}^{\prime}, R_{l}^{\prime \prime}, R_{-2, l}\right)=\varphi\left(R_{2}^{\prime}, R_{l}^{\prime}, R_{-2, l}\right)$. But $c^{\prime}$ is not a top cycle for any strict transformation of $\left(R_{2}^{\prime}, R_{l}^{\prime \prime}, R_{-2, l}\right)$ because $1, j \in C^{\prime}$, which is a contradiction to Lemma 2. Thus, $\varphi_{l}\left(R_{2}^{\prime}, R_{l}^{\prime \prime}, R_{-2, l}\right)=1$.

By individual rationality and construction, for all $i \in C^{\prime} \backslash\{1, l\}, \varphi_{i}\left(R_{2}^{\prime}, R_{l}^{\prime \prime}, R_{-2, l}\right)=i$. Now by consistency, $\varphi_{l}\left(\left.\left(R_{1}, R_{2}^{\prime}, R_{l}^{\prime \prime}\right)\right|_{\{1,2, l\}}\right)=1$. Let $R_{l}^{\prime \prime \prime}: 1, l, 2$. By strategy-proofness, $\varphi_{l}\left(\left.\left(R_{1}, R_{2}^{\prime}\right)\right|_{\{1,2, l\}}, R_{l}^{\prime \prime \prime}\right)=1$. Note that profile $\left(\left.\left(R_{1}, R_{2}^{\prime}\right)\right|_{\{1,2, l\}}, R_{l}^{\prime \prime \prime}\right)$ is identical to the profile in (1) where $i=1, j=2$, and $k=l$ (because $2 I_{1} l$ and $2 P_{1} 1$ ). Now this contradicts the definition $2 \succ_{1} l$ (because $(2,1)$ is a top cycle in $f^{\succ}(R)$ and $l \in t o p\left(R_{1}\right)$, we cannot have $l \succ_{1} 2$ ).

Let $k \geq 3$ and suppose that $3 P_{2} \varphi_{2}(R)$. Let $R_{2}^{\prime}: 3,2, \ldots$ By strategy-proofness and $\varphi_{2}(R) \neq 3$, we have $\varphi_{2}\left(R_{2}^{\prime}, R_{-2}\right) \neq 3$. By individual rationality, $\varphi_{2}\left(R_{2}^{\prime}, R_{-2}\right)=2$.

Note that $c$ remains a top cycle under $\left(R_{2}^{\prime}, R_{-2}\right)$ and $f_{2}^{\succ}\left(R_{2}^{\prime}, R_{-2}\right)=3$. Thus, without loss of generality we may suppose that for all $i \in\{1, \ldots, k\}$, if $f_{i}^{\succ}(R) P_{i} \varphi_{i}(R)$, then $R_{i}: f_{i}^{\succ}(R), i, \ldots$

Furthermore, for all $i \in\{1, \ldots, k\}$ such that $\varphi_{i}(R)=f_{i}^{\succ}(R)$, by strategy-proofness and nonbossiness we may assume without loss of generality that $R_{i}: \varphi_{i}(R), i, \ldots$

Now if for all $i \in\{1, \ldots, k\},\left|\operatorname{top}\left(R_{i}\right)\right|=1$, then by construction and individual rationality, $\varphi_{i}(R)=i$ for all $\{1, \ldots, k\}$. Now this contradicts consistency and weak efficiency.

Thus, there exists some $i \in\{1, \ldots, k\}$ such that $\left|\operatorname{top}\left(R_{i}\right)\right| \geq 2$ and therefore, $\varphi_{i}(R) \in \operatorname{top}\left(R_{i}\right)$ and $\varphi_{i}(R) \neq f_{i}^{\succ}(R)$, say $i=1$.

First, suppose that agent 1 is the only agent $i \in\{1 \ldots, k\}$ with $\left|\operatorname{top}\left(R_{i}\right)\right| \geq 2$. Note that because $c$ is a top cycle in $f^{\succ}(R)$, we have $2 \succ_{1} j$ for all $j \in \operatorname{top}\left(R_{1}\right) \backslash\{2\}$. Also note that $1 \notin \operatorname{top}\left(R_{1}\right)$. Now also by strategy-proofness and non-bossiness, without loss of generality, we may suppose that for all $i \in N \backslash\{1, \ldots, k\}, R_{i}: \varphi_{i}(R), i, \ldots$ (if $\varphi_{i}(R) \neq i$ ) and $R_{i}: i, \ldots$ (if $\varphi_{i}(R)=i$ ).

Let $\varphi_{1}(R)=l$. Note that $l \neq 2$ and $2 \succ_{1} l$. Let $R_{2}^{\prime}: 3,1,2, \ldots$ Now note that under $\left(R_{2}^{\prime}, R_{-2}\right)$ the cycle $(2,1)$ is not a top cycle for any strict transformation of $R$ and for all $i \in N \backslash\{1,2\}$, $i P_{i} 2$. Thus, by Lemma $2, \varphi_{2}\left(R_{2}^{\prime}, R_{-2}\right) \neq 1$. Now by individual rationality and strategy-proofness, $\varphi_{2}\left(R_{2}^{\prime}, R_{-2}\right)=2$. By non-bossiness, $\varphi\left(R_{2}^{\prime}, R_{-2}\right)=\varphi(R)$. Let $R_{2}^{\prime \prime}: 1,2, \ldots$ Again by individual rationality, strategy-proofness and non-bossiness, we obtain $\varphi\left(R_{2}^{\prime \prime}, R_{-2}\right)=\varphi(R)$. Now $(2,1)$ is a top cycle in $f^{\succ}\left(R_{2}^{\prime \prime}, R_{-2}\right)$ of length two. Above we have shown that Lemma 4 is true for top cycle of length two, i.e. we must have $\varphi_{2}\left(R_{2}^{\prime \prime}, R_{-2}\right) I_{2} 1$. This contradicts the fact that $\varphi_{2}\left(R_{2}^{\prime \prime}, R_{-2}\right)=2$ 
and $1 P_{2}^{\prime \prime} 2$.

Second, suppose that 1 is not the only agent $i \in\{1 \ldots, k\}$ with $\left|\operatorname{top}\left(R_{i}\right)\right| \geq 2$. Recall that for any $i \in\{1, \ldots, k\}$ such that $\left|\operatorname{top}\left(R_{i}\right)\right| \geq 2$ we have $\varphi_{i}(R) \in \operatorname{top}\left(R_{i}\right)$ and $\varphi_{i}(R) \neq f_{i}^{\succ}(R)$. Let $m$ be the number of such agents in the cycle $c$. Above we have shown that the induction basis holds for $m=1$. By induction, now we may suppose $\varphi_{i}(R) I_{i} f_{i}^{\succ}(R)$ for all $i \in\{1, \ldots, k\}$ if the cycle $c$ contains $m$ agents or fewer with $\left|\operatorname{top}\left(R_{i}\right)\right| \geq 2$.

Now suppose that we do not have $\varphi_{i}(R) I_{i} f_{i}^{\succ}(R)$ for all $i \in\{1, \ldots, k\}$ and there are $m+1$ agents in the cycle $c$ with $\left|\operatorname{top}\left(R_{i}\right)\right| \geq 2$. Note that for any $i \in\{1, \ldots, k\}$ such that $f_{i}^{\succ}(R) P_{i} \varphi_{i}(R)$ we have $\varphi_{i}(R)=i$. Now if $\left|\operatorname{top}\left(R_{i-1}\right)\right| \geq 2$, then $\varphi_{i-1}(R) \in \operatorname{top}\left(R_{i-1}\right)$. Otherwise $\operatorname{top}\left(R_{i-1}\right)=\{i\}$ and $\varphi_{i-1}(R)=i-1$. Thus, starting from $i$ we will find eventually an agent $j$ such that $\left|\operatorname{top}\left(R_{j}\right)\right| \geq 2$.

Now consider again agent 1 and agent 2 as above. Note that $\varphi_{1}(R) \neq 2$ and $\varphi_{2}(R)=2$. Let $R_{2}^{\prime}: 1,2, \ldots$. Now $(2,1)$ is a top cycle under $f^{\succ}\left(R_{2}^{\prime}, R_{-2}\right)$ and only for agent 1 we have $\left|\operatorname{top}\left(R_{1}\right)\right| \geq 2$. Now by the induction hypothesis, we must have $\varphi_{2}\left(R_{2}^{\prime}, R_{-2}\right)=1$. Consider $R_{2}^{\prime \prime}: 3,1,2, \ldots$ Now by strategy-proofness, we must have $\varphi_{2}\left(R_{2}^{\prime \prime}, R_{-2}\right)=1$. By Lemma 2 , agent 3 must trade before agent 2. Note that $c$ remains a top cycle under $f^{\succ}\left(R_{2}^{\prime \prime}, R_{-2}\right)$.

Now if $\varphi_{1}\left(R_{2}^{\prime \prime}, R_{-2}\right)=2$, then let $R_{1}^{\prime}: 2,1, \ldots$ By strategy-proofness and non-bossiness, $\varphi\left(R_{1}^{\prime}, R_{2}^{\prime \prime}, R_{-1,2}\right)=\varphi\left(R_{2}^{\prime \prime}, R_{-2}\right)$. Again by strategy-proofness, $\varphi_{2}\left(R_{1}^{\prime}, R_{2}^{\prime \prime}, R_{-2}\right)=1$, and $R_{2}^{\prime \prime}$ : $3,1,2, \ldots$, we have $\varphi_{2}\left(R_{1}^{\prime}, R_{-1}\right)=2$. Note that $c$ is a top cycle under $f^{\succ}\left(R_{1}^{\prime}, R_{-1}\right)$. But now for the top cycle $c$ we have $\left|\operatorname{top}\left(R_{1}^{\prime}\right)\right|=1$ meaning that under the profile $\left(R_{1}^{\prime}, R_{-1}\right)$ there are $m$ agents $i$ with $\left|\operatorname{top}\left(R_{i}\right)\right| \geq 2$. By the induction hypothesis, we now must have $\varphi_{2}\left(R_{1}^{\prime}, R_{-1}\right) I_{2} f_{2}^{\succ}\left(R_{1}^{\prime}, R_{-1}\right)$. Since $\operatorname{top}\left(R_{2}\right)=\{3\}$, this contradicts $\varphi_{2}\left(R_{1}^{\prime}, R_{-1}\right)=2$.

Thus, $\varphi_{1}\left(R_{2}^{\prime \prime}, R_{-2}\right) \neq 2$, say $\varphi_{1}\left(R_{2}^{\prime \prime}, R_{-2}\right)=l$. Let $c^{\prime \prime}$ denote the cycle along which 1 trades in $\varphi\left(R_{2}^{\prime \prime}, R_{-2}\right)$, and let $C^{\prime \prime}$ be this coalition. Note that $1,2 \in C^{\prime \prime}$ and $\left|C^{\prime \prime}\right| \geq 3$ because $l \neq 2$. Let $\hat{R}_{C^{\prime \prime}}=\left.\left(R_{2}^{\prime \prime}, R_{C^{\prime \prime} \backslash\{2\}}\right)\right|_{C^{\prime \prime}}$. By consistency, $\varphi_{i}\left(\hat{R}_{C^{\prime \prime}}\right)=\varphi_{i}\left(R_{2}^{\prime \prime}, R_{-2}\right)$ for all $i \in C^{\prime \prime}$. If $3 \in C^{\prime \prime}$, then by $\hat{R}_{2}: 3,1,2, \ldots$ the cycle $c^{\prime \prime}$ is not a top cycle for any strict transformation of $\hat{R}_{C^{\prime \prime}}$, which would contradict Lemma 2.

Because $c$ was a top cycle under $f^{\succ}(R)$, we have $2 \succ_{1} l$. But now $(2,1)$ is a top cycle under $f^{\succ}\left(\hat{R}_{C^{\prime \prime}}\right)$ and both $\left|\operatorname{top}\left(\hat{R}_{1}\right)\right| \geq 2$ and $\operatorname{top}\left(\hat{R}_{2}\right)=\{1\}$ (by $\left.3 \notin C^{\prime \prime}\right)$. Note that $c^{\prime \prime}$ may still be a top cycle for some strict transformation of $\hat{R}_{C^{\prime \prime}}$.

Now by strategy-proofness and non-bossiness, we may assume without loss of generality that for all $i \in C^{\prime \prime} \backslash\{1,2\}, \hat{R}_{i}: \varphi_{i}\left(\hat{R}_{C^{\prime \prime}}\right), i, \ldots$ Let $R_{l}^{\prime}: 1, \varphi_{l}\left(\hat{R}_{C^{\prime \prime}}\right), l, \ldots$ Now by strategy-proofness, $\varphi_{l}\left(R_{l}^{\prime}, \hat{R}_{C^{\prime \prime} \backslash\{l\}}\right) \in\left\{1, \varphi_{l}\left(\hat{R}_{C^{\prime \prime}}\right)\right\}$. Note that $c^{\prime \prime}$ is not a top cycle for any strict transformation of $\left(R_{l}^{\prime}, \hat{R}_{C^{\prime \prime} \backslash\{l\}}\right)$. Hence, by construction, $\varphi_{l}\left(R_{l}^{\prime}, \hat{R}_{C^{\prime \prime} \backslash\{l\}}\right)=1$. By individual rationality and $3 \notin C^{\prime \prime}$, $\varphi_{2}\left(R_{l}^{\prime}, \hat{R}_{C^{\prime \prime} \backslash\{l\}}\right)=2$. Now this is a contradiction to our induction hypothesis because $(2,1)$ is a top cycle under $f^{\succ}\left(R_{l}^{\prime}, \hat{R}_{C^{\prime \prime} \backslash\{l\}}\right)$ but $f_{2}^{\succ}\left(R_{l}^{\prime}, \hat{R}_{C^{\prime \prime} \backslash\{l\}}\right) P_{2} \varphi_{2}\left(R_{l}^{\prime}, \hat{R}_{C^{\prime \prime} \backslash\{l\}}\right)$.

Therefore, we have shown by induction that for any top cycle $c$ with coalition $C$ in $f^{\succ}(R)$, we have $\varphi_{i}(R) I_{i} f^{\succ}(R)$ for all $i \in C$. 
The following is the second key to the proof of Theorem 1. We show that for any top cycle in $f^{\succ}(R)$ the members of its trading coalition cannot be assigned any object of an agent who does not belong to the trading coalition.

Lemma 5. For all $R \in \mathcal{R}^{N}$, if $c$ is a top cycle in $f^{\succ}(R)$ with trading coalition $C$, then $\cup_{i \in C}\left\{\varphi_{i}(R)\right\}=C$.

Proof. Let $R \in \mathcal{R}^{N}$ and let $c$ be a top cycle in $f^{\succ}(R)$ with trading coalition $C$. Without loss of generality, let $C=\{1, \ldots, k\}$ and $c=(2,3, \ldots, k, 1)$ (where 1 receives 2,2 receives $3, \ldots, k$ receives 1). By Lemma 4 , for all $i \in\{1, \ldots, k\}, f_{i}^{\succ}(R) I_{i} \varphi_{i}(R)$. We show that $\varphi_{i}(R) \in\{1, \ldots, k\}$ for all $i \in\{1, \ldots, k\}$.

If $k=1$, then by individual rationality and the fact that there is no $i \neq 1$ such that $i I_{1} 1$, $\varphi_{1}(R)=1=f_{1}^{\succ}(R)$, the desired conclusion.

Let $k \geq 2$. If for all $i \in\{1, \ldots, k\},\left|\operatorname{top}\left(R_{i}\right)\right|=1$, then by Lemma $4, \varphi_{i}(R)=f_{i}^{\succ}(R)$ for all $i \in\{1, \ldots, k\}$, the desired conclusion.

We now show that Lemma 5 is true for the cycle $c$ if there is at most one agent in the cycle whose set of most preferred objects contains more than one element.

Claim 1: Suppose that for exactly one $l \in\{1, \ldots, k\}$ we have $\left|\operatorname{top}\left(R_{l}\right)\right| \geq 2$ and for all $j \in$ $\{1, \ldots, k\} \backslash\{l\}$ we have $\left|\operatorname{top}\left(R_{j}\right)\right|=1$. Then for all $i \in\{1, \ldots, k\}, \varphi_{i}(R)=f_{i}^{\succ}(R)$.

Proof of Claim 1: Without loss of generality, let $\left|\operatorname{top}\left(R_{1}\right)\right| \geq 2$. Now by Lemma 4, we have for all $i \in\{2, \ldots, k\}, \varphi_{i}(R)=f_{i}^{\succ}(R)$ (because $\left.\operatorname{top}\left(R_{i}\right)=\left\{f_{i}^{\succ}(R)\right\}\right)$. We need to show $\varphi_{1}(R)=2$. By Lemma $4, \varphi_{1}(R) \in \operatorname{top}\left(R_{1}\right)$. Let $\varphi_{1}(R)=l$. Suppose that $l \neq 2$. Now we must have $l \notin\{1, \ldots, k\}$. Let $c^{\prime}$ be the cycle along which agent 1 trades in $\varphi(R)$ with coalition $C^{\prime}$. By $\varphi_{k}(R)=1$, we have $\{1, \ldots, k\} \subseteq C^{\prime}$. Let $R_{l}^{\prime}: 2, \varphi_{l}(R), l, \ldots$ By strategy-proofness, $\varphi_{l}\left(R_{l}^{\prime}, R_{-l}\right) \neq l$. If $\varphi_{l}\left(R_{l}^{\prime}, R_{-l}\right)=$ $\varphi_{l}(R)$, then by non-bossiness, $\varphi\left(R_{l}^{\prime}, R_{-l}\right)=\varphi(R)$. But now $c^{\prime}$ is not a top cycle for any strict transformation of $\left(R_{l}^{\prime}, R_{-l}\right)$ because $1, l \in C^{\prime}$ and $1 P_{l}^{\prime} \varphi_{l}(R)$. Thus, $\varphi_{l}\left(R_{l}^{\prime}, R_{-l}\right)=2$. But now $\varphi_{2}\left(R_{l}^{\prime}, R_{-l}\right) \neq 2$ and by $\left|\operatorname{top}\left(R_{2}\right)\right|=\left\{f_{2}^{\succ}(R)\right\}$, we have $\varphi_{2}\left(R_{l}^{\prime}, R_{-l}\right)=f_{2}^{\succ}(R)$. Similarly, we have $\varphi_{i}\left(R_{l}^{\prime}, R_{-l}\right)=f_{i}^{\succ}(R)$ for all $i \in\{2, \ldots, k\}$. In particular, the agents $l, 1,2, \ldots, k$ belong to the same trading cycle in $\varphi\left(R_{l}^{\prime}, R_{-l}\right)$. Let $c^{\prime \prime}$ denote this cycle with coalition $C^{\prime \prime}$. Let $R_{l}^{\prime \prime}: 1,2, l, \ldots$ Now if $\varphi_{l}\left(R_{l}^{\prime \prime}, R_{-l}\right)=2$, then by non-bossiness, $\varphi\left(R_{l}^{\prime \prime}, R_{-l}\right)=\varphi\left(R_{l}^{\prime}, R_{-l}\right)$ which contradicts Lemma 2 since $c^{\prime \prime}$ is not a top cycle for any strict transformation of $\left(R_{l}^{\prime \prime}, R_{-l}\right)$. Now by strategy-proofness, $\varphi_{l}\left(R_{l}^{\prime \prime}, R_{-l}\right)=1$. By $\operatorname{top}\left(R_{k}\right)=\{1\}$, we now have $\varphi_{k}\left(R_{l}^{\prime \prime}, R_{-l}\right)=k$. Since $c$ remains a top cycle under $f^{\succ}\left(R_{l}^{\prime \prime}, R_{-l}\right)$, this is a contradiction to Lemma 4 .

Suppose that $k=2$ and both $\left|\operatorname{top}\left(R_{1}\right)\right| \geq 2$ and $\operatorname{top}\left(R_{2}\right)=\{1\}$. By Lemma $4, \varphi_{2}(R)=1$. By Claim $1, \varphi_{1}(R)=2$.

Finally suppose that both $\left|\operatorname{top}\left(R_{1}\right)\right| \geq 2$ and $\left|\operatorname{top}\left(R_{2}\right)\right| \geq 2$. Now if $\varphi_{1}(R)=2$ or $\varphi_{2}(R)=1$, then we obtain from strategy-proofness and non-bossiness and Claim 1 the conclusion. 
Thus, suppose that $\varphi_{1}(R) \neq 2$ and $\varphi_{2}(R) \neq 1$. Let $c^{1}$ and $c^{2}$ denote the trading cycles for 1 and 2 with corresponding coalitions $C^{1}$ and $C^{2}$. By consistency, we may suppose without loss of generality $C^{1} \cup C^{2}=N$. By strategy-proofness and non-bossiness, we may suppose that for all $i \in N \backslash\{1,2\}, R_{i}: \varphi_{i}(R), i, \ldots$ Let $\varphi_{1}(R)=l$ and $R_{l}^{\prime}: 1, \varphi_{l}(R), l, \ldots$ If $\varphi_{l}\left(R_{l}^{\prime}, R_{-l}\right)=\varphi_{l}(R)$, then $\varphi\left(R_{l}^{\prime}, R_{-l}\right)=\varphi(R)$. Since $1, l \in C^{1}$, then $c^{1}$ is not a top cycle for any strict transformation of $\left(R_{l}^{\prime}, R_{-l}\right)$ (even after executing $\left.c^{2}\right)$, a contradiction to Lemma 2 . Thus, $\varphi_{l}\left(R_{l}^{\prime}, R_{-l}\right)=1$. Now for any $i \in C^{1} \backslash\{1, l\}$, if $R_{i}: 1, i, \ldots$, then $\varphi_{i}\left(R_{l}^{\prime}, R_{-l}\right)=i$ and we may apply consistency in order to remove agent $i$ from the economy.

Now if $\varphi_{1}\left(R_{l}^{\prime}, R_{-l}\right)=2$, then we can use strategy-proofness and non-bossiness to derive a contradiction using Claim 1.

Thus, $\varphi_{1}\left(R_{l}^{\prime}, R_{-l}\right) \neq 2$. Let $R_{l}^{\prime \prime}: 2,1, l, \ldots$ Suppose that $\varphi_{l}\left(R_{l}^{\prime \prime}, R_{-l}\right)=2$. Then $\varphi_{2}\left(R_{l}^{\prime \prime}, R_{-l}\right)=$ 1 because agent 2 is the only agent $i$ such that $1 P_{i} i$ and agent 1 cannot receive 1 . Now again we can derive a contradiction using Claim 1. But now by construction and individual rationality, object 1 can be only assigned to agent 1 , a contradiction to Lemma 4.

Hence, $\varphi_{l}\left(R_{l}^{\prime \prime}, R_{-l}\right)=1$ and by non-bossiness, $\varphi\left(R_{l}^{\prime \prime}, R_{-l}\right)=\varphi\left(R_{l}^{\prime}, R_{-l}\right)$. Note that by $R_{l}^{\prime \prime}$ : $2,1, l, \ldots$ agents $l$ and 2 cannot be part of the same trading cycle. Thus, $\varphi_{1}\left(R_{l}^{\prime \prime}, R_{-l}\right)=l$ and again $c^{2}$ is a trading cycle under $\varphi\left(R_{l}^{\prime \prime}, R_{-l}\right)$.

Let $j \in N$ be such that $\varphi_{j}\left(R_{l}^{\prime \prime}, R_{-l}\right)=2$. Note that $j \notin\{l, 1,2\}$. Let $R_{j}^{\prime}: l, 2, j, \ldots$.

First, suppose that $\varphi_{j}\left(R_{l}^{\prime \prime}, R_{j}^{\prime}, R_{-j, l}\right)=2$. By non-bossiness, $\varphi\left(R_{l}^{\prime \prime}, R_{j}^{\prime}, R_{-j, l}\right)=\varphi\left(R_{l}^{\prime \prime}, R_{-l}\right)$. Now neither $c^{2}$ nor $(l, 1)$ is a top cycle for any strict transformation of $\left(R_{l}^{\prime \prime}, R_{j}^{\prime}, R_{-j, l}\right)$. This is a contradiction to Lemma 2. Thus, $\varphi_{j}\left(R_{l}^{\prime \prime}, R_{j}^{\prime}, R_{-j, l}\right)=l$. Then $\varphi_{2}\left(R_{l}^{\prime \prime}, R_{j}^{\prime}, R_{-j, l}\right)=\varphi_{2}\left(R_{l}^{\prime \prime}, R_{-l}\right)$. Also again $\varphi_{1}\left(R_{l}^{\prime \prime}, R_{j}^{\prime}, R_{-j, l}\right) \neq 2$ because otherwise we apply again the previous fact. Thus, $\varphi_{l}\left(R_{l}^{\prime \prime}, R_{j}^{\prime}, R_{-j, l}\right)=2$. But then $\varphi_{1}\left(R_{l}^{\prime \prime}, R_{j}^{\prime}, R_{-j, l}\right)=1$, a contradiction to Lemma 4 .

Now let $k \geq 3$. We make the following observation: by consistency, we may suppose that for all $i \in N \backslash\{1, \ldots, k\}, \varphi_{i}(R) \neq i$ (and the trading cycle $c^{i}$ with coalition $i \in C^{i}$ we have $\left.C^{i} \cap\{1, \ldots, k\} \neq \emptyset\right)$ and $R_{i}: \varphi_{i}(R), i, \ldots$ Furthermore, by strategy-proofness and non-bossiness we may suppose that for all $i \in N \backslash\{1, \ldots, k\}, \varphi_{i}(R) \in\{1, \ldots, k\}$; suppose not, say there exists $i \in N \backslash\{1, \ldots, k\}$ such that $\varphi_{i}(R) \notin\{1, \ldots, k\}$. Note that $C^{i} \cap\{1, \ldots, k\} \neq \emptyset$. Without loss of generality, $1 \in C^{i}$ and $\varphi_{1}(R)=i$. Let $R_{i}^{\prime}: 1, \varphi_{i}(R), i, \ldots$ If $\varphi_{i}\left(R_{i}^{\prime}, R_{-i}\right)=\varphi_{i}(R)$, then by strategy-proofness and non-bossiness, $\varphi\left(R_{i}^{\prime}, R_{-i}\right)=\varphi(R)$. Since $1, i \in C^{i}, c^{i}$ is not a top cycle for any strict transformation of $\left(R_{i}^{\prime}, R_{-i}\right)$, a contradiction to Lemma 2. Thus, $\varphi_{i}\left(R_{i}^{\prime}, R_{-i}\right)=1$. Let $R_{i}^{\prime \prime}: 1, i, \ldots$ By strategy-proofness and non-bossiness, $\varphi\left(R_{i}^{\prime \prime}, R_{-i}\right)=\varphi\left(R_{i}^{\prime}, R_{-i}\right)$. Now again for some $j \in\{1, \ldots, k\}, \varphi_{j}\left(R_{i}^{\prime \prime}, R_{-i}\right) \notin\{1, \ldots, k\}$, and $c$ remains a top cycle in $f^{\succ}\left(R_{i}^{\prime \prime}, R_{-i}\right)$. Thus, without loss of generality we may suppose that already $R_{i}: 1, i, \ldots$

We prove Lemma 5 by induction on the number of agents whose set of most preferred objects contains two or more elements.

Induction basis: For any top cycle $c$ in $f^{\succ}(R)$ with trading coalition $C$ (where $|C| \geq 3$ ), if there 
exists some $l \in C$ such that for all $j \in C \backslash\{l\},\left|\operatorname{top}\left(R_{j}\right)\right|=1$, then for all $i \in C, \varphi_{i}(R) \in C$.

Obviously, Claim 1 establishes the induction basis.

Induction hypothesis: For any top cycle $c$ in $f^{\succ}(R)$ with trading coalition $C$ (where $|C| \geq 3$ ), if there exist $l_{1}, \ldots, l_{m} \in C$ such that for all $j \in C \backslash\left\{l_{1}, \ldots, l_{m}\right\},\left|\operatorname{top}\left(R_{j}\right)\right|=1$, then for all $i \in C$, $\varphi_{i}(R) \in C$.

Using the induction hypothesis we will prove the induction step. Again consider $c=$ $(2,3 \ldots, k, 1)$ and suppose that there exist $l_{1}, \ldots, l_{m+1} \in\{1, \ldots, k\}$ such that for all $j \in$ $\{1, \ldots, k\} \backslash\left\{l_{1}, \ldots, l_{m+1}\right\},\left|\operatorname{top}\left(R_{j}\right)\right|=1$.

Suppose that for some $i \in\{1, \ldots, k\}, \varphi_{i}(R) \notin\{1, \ldots, k\}$, say $\varphi_{1}(R)=l \notin\{1, \ldots, k\}$. Now $2, l \in \operatorname{top}\left(R_{1}\right)$ and $\left|\operatorname{top}\left(R_{1}\right)\right| \geq 2$. By strategy-proofness and non-bossiness, we may assume without loss of generality that for all $i \in N \backslash\{1, \ldots, k\}, R_{i}: \varphi_{i}(R), i, \ldots$

Let $R_{l}^{\prime}: 1, \varphi_{l}(R), l, \ldots$. Since 1 and $l$ belong to the same cycle in $\varphi(R)$, similarly as in the proof of Claim 1, we cannot have $\varphi_{l}\left(R_{l}^{\prime}, R_{-l}\right)=\varphi_{l}(R)$. Thus, by strategy-proofness, $\varphi_{l}\left(R_{l}^{\prime}, R_{-l}\right)=1$. But now $\varphi_{k}\left(R_{l}^{\prime}, R_{-l}\right) \neq 1$ and by Lemma 4 (since $c$ remains a top cycle under $f^{\succ}\left(R_{l}^{\prime}, R_{-l}\right)$ ), $\varphi_{k}\left(R_{l}^{\prime}, R_{-l}\right) \in \operatorname{top}\left(R_{k}\right)$. Thus, $\left|\operatorname{top}\left(R_{k}\right)\right| \geq 2$.

Now we have for all $i \in\{2, \ldots, k\}, \varphi_{i}(R) \neq 1$ : otherwise, say for $i \in\{2, \ldots, k\}$, we have $\varphi_{i}(R)=1$; now for $i<k$ we have $1, i+1 \in \operatorname{top}\left(R_{i}\right)$ and for $i=k$ we have $\left|\operatorname{top}\left(R_{k}\right)\right| \geq 2$. Now let $R_{i}^{\prime}: 1, i, \ldots$ By strategy-proofness and non-bossiness, we have $\varphi\left(R_{i}^{\prime}, R_{-i}\right)=\varphi(R)$. Note that $(2,3, \ldots, i, 1)$ is a top cycle under $f^{\succ}\left(R_{i}^{\prime}, R_{-i}\right)$. Since $\varphi_{1}\left(R_{i}^{\prime}, R_{-i}\right)=l$ and $l \notin C$, this now contradicts our induction hypothesis because $\operatorname{top}\left(R_{i}^{\prime}\right)=\{1\}$ and for the cycle $(2,3, \ldots, i, 1)$ there are $m$ or fewer agents $j \in\{1, \ldots, i-1\}$ with $\left|\operatorname{top}\left(R_{j}\right)\right| \geq 2$.

If $\varphi_{1}\left(R_{l}^{\prime}, R_{-l}\right)=2$, then we can use strategy-proofness, non-bossiness, and the induction hypothesis to derive a contradiction. Thus, $\varphi_{1}\left(R_{l}^{\prime}, R_{-l}\right) \neq 2$.

Furthermore, by $\varphi_{l}\left(R_{l}^{\prime}, R_{-l}\right)=1$, for all $i \in N \backslash\{1, \ldots, k\}, \varphi_{i}\left(R_{l}^{\prime}, R_{-l}\right) \neq 1$. Thus, if $R_{i}: 1, i, \ldots$, then we may remove $i$ using consistency without changing the allocation for the other agents. Thus, we may assume that there exist no $i \in N \backslash\{l, 1,2, \ldots, k\}$ such that $R_{i}: 1, i, \ldots$

Let $R_{l}^{\prime \prime}: 2,1, l, \ldots$. We show that $\left|\operatorname{top}\left(R_{2}\right)\right| \geq 2$ and $\varphi_{2}\left(R_{l}^{\prime \prime}, R_{-l}\right) \notin C$. First, suppose that $\varphi_{l}\left(R_{l}^{\prime \prime}, R_{-l}\right)=2$. Since $i P_{i} 1$ for all $i \in N \backslash\{l, 1,2, \ldots, k\}$, there exists some $j \in\{2, \ldots, k\}$ such that $\varphi_{j}\left(R_{l}^{\prime \prime}, R_{-l}\right)=1$. Now if $j<k$, then $j+1,1 \in \operatorname{top}\left(R_{j}\right)$ and $\left|\operatorname{top}\left(R_{j}\right)\right| \geq 2$, and if $j=k$, then $\left|\operatorname{top}\left(R_{k}\right)\right| \geq 2$. Let $R_{j}^{\prime}: 1, j, \ldots$ By strategy-proofness and non-bossiness, $\varphi\left(R_{l}^{\prime \prime}, R_{j}^{\prime}, R_{-l, j}\right)=$ $\varphi\left(R_{l}^{\prime \prime}, R_{-l}\right)$. Now $(2,3, \ldots, j, 1)$ is a top cycle in $f^{\succ}\left(R_{l}^{\prime \prime}, R_{j}^{\prime}, R_{-l}\right)$ and the induction hypothesis applies. This is a contradiction to $\varphi_{l}\left(R_{l}^{\prime \prime}, R_{j}^{\prime}, R_{-l, j}\right)=2$ (and $\left.l \notin\{1,2, \ldots, j\}\right)$.

Thus, $\varphi_{l}\left(R_{l}^{\prime \prime}, R_{-l}\right)=1$. Next we show that we may assume without loss of generality that $\varphi_{1}\left(R_{l}^{\prime \prime}, R_{-l}\right)=l$. Again $\varphi_{1}\left(R_{l}^{\prime \prime}, R_{-l}\right) \notin\{2, \ldots, k\}$ because otherwise we use the induction hypothesis to derive a contradiction. Suppose that $\varphi_{1}\left(R_{l}^{\prime \prime}, R_{-l}\right)=h \notin\{l, 1, \ldots, k\}$. Let $\tilde{R}_{l}: 1, l, \ldots$ By strategy-proofness and non-bossiness, $\varphi\left(\tilde{R}_{l}, R_{-l}\right)=\varphi\left(R_{l}^{\prime \prime}, R_{-l}\right)$. Let $R_{h}^{\prime}: 1, \varphi_{h}\left(\tilde{R}_{l}, R_{-l}\right), h, \ldots$ As above we obtain $\varphi_{h}\left(\tilde{R}_{l}, R_{h}^{\prime}, R_{-l, h}\right)=1$. By individual rationality, $\varphi_{l}\left(\tilde{R}_{l}, R_{h}^{\prime}, R_{-l, h}\right)=l$. Now again 
$\varphi_{1}\left(\tilde{R}_{l}, R_{h}^{\prime}, R_{-l, h}\right) \notin\{1,2, \ldots, k\}$. By consistency, we may remove $l$ from the economy $\left(\tilde{R}_{l}, R_{h}^{\prime}, R_{-l, h}\right)$ without changing the allocation. Since $N \backslash\{1, \ldots, k\}$ is finite, we eventually need to have that agent 1 receives the endowment of the agent who is receiving object 1 .

Thus, $\varphi_{1}\left(R_{l}^{\prime \prime}, R_{-l}\right)=l$. Similarly as above, we cannot have $\varphi_{2}\left(R_{l}^{\prime \prime}, R_{-l}\right) \in\{1,4, \ldots, k\}$. Suppose that $\varphi_{2}\left(R_{l}^{\prime \prime}, R_{-l}\right)=3$. But now $\operatorname{top}\left(R_{2}\right)=\{3\}$ because otherwise we obtain a contradiction using the induction hypothesis. Now take the first agent $j \in\{3,4, \ldots, k\}$ such that $\left|\operatorname{top}\left(R_{j}\right)\right| \geq 2$. Again $\varphi_{j}\left(R_{l}^{\prime \prime}, R_{-l}\right) \notin\{j+1, \ldots, k\}$.

Thus, $\varphi_{j}\left(R_{l}^{\prime \prime}, R_{-l}\right)=h \notin\{1, \ldots, k\}$. But now for $j$ in the role of 1 and $h$ in the role of $l$ we would obtain for $R_{h}^{\prime}: j, \varphi_{h}\left(R_{l}^{\prime \prime}, R_{-l}\right), h, \ldots$ that $\varphi_{h}\left(R_{l}^{\prime \prime}, R_{h}^{\prime}, R_{-l, h}\right)=j$. But now by $\operatorname{top}\left(R_{j-1}\right)=\{j\}$ we now have $\varphi_{j-1}\left(R_{l}^{\prime \prime}, R_{h}^{\prime}, R_{-l, h}\right)=j-1$. Since $c$ remains a top cycle for $f^{\succ}\left(R_{l}^{\prime \prime}, R_{h}^{\prime}, R_{-l, h}\right)$, this is a contradiction to Lemma 4 .

Hence, $\varphi_{2}\left(R_{l}^{\prime \prime}, R_{-l}\right) \notin\{1, \ldots, k\}$ and $\left|\operatorname{top}\left(R_{2}\right)\right| \geq 2$. Now using the same arguments it follows that $\left|\operatorname{top}\left(R_{3}\right)\right| \geq 2$ and so on. Hence, $\left|\operatorname{top}\left(R_{i}\right)\right| \geq 2$ for all $i \in\{1, \ldots, k\}$.

But now again as above, $\varphi_{i}\left(R_{l}^{\prime \prime}, R_{-l}\right) \notin\{1, \ldots, k\}$ for all $i \in\{1, \ldots, k\}$ : we know already $\varphi_{2}\left(R_{l}^{\prime \prime}, R_{-l}\right) \notin\{1, \ldots, k\}$; if $\varphi_{3}\left(R_{l}^{\prime \prime}, R_{-l}\right)=t \in\{1, \ldots, k\}$, then let $R_{3}^{\prime}: t, 3, \ldots$ By strategyproofness and non-bossiness, $\varphi\left(R_{l}^{\prime \prime}, R_{3}^{\prime}, R_{-l, 3}\right)=\varphi\left(R_{l}^{\prime \prime}, R_{-3}\right)$. But now $(2,3, t, t+1, \ldots, k, 1)$ (we may have $t=2)$ is a top cycle in $f^{\succ}\left(R_{l}^{\prime \prime}, R_{3}^{\prime}, R_{-l, 3}\right)$ and the induction hypothesis applies. However, this contradicts $\varphi_{2}\left(R_{l}^{\prime \prime}, R_{3}^{\prime}, R_{-l}\right) \notin\{1, \ldots, k\}$. The same applies for agent 4 and so on.

For all $i \in\{1, \ldots, k\}$, let $\varphi_{i}\left(R_{l}^{\prime \prime}, R_{-l}\right)=h_{i} \notin\{1, \ldots, k\}$. Note that $\varphi_{l}\left(R_{l}^{\prime \prime}, R_{-l}\right)=1$ and for all $i \in N \backslash\{l, 1,2 \ldots, k\}$, there exists $t \in\{1, \ldots, k\}$ such that $R_{i}: t, i, \ldots$ Now $\varphi_{i}\left(R_{l}^{\prime \prime}, R_{-l}\right) \neq i$ for all $i \in N$ because otherwise any such agent may be removed using consistency. Because $\varphi_{i}\left(R_{l}^{\prime \prime}, R_{-l}\right) \notin\{1, \ldots, k\}$ for all $i \in\{1, \ldots, k\}$ and $\varphi_{i}\left(R_{l}^{\prime \prime}, R_{-l}\right) \in\{1, \ldots, k\}$ for all $i \in N \backslash\{1, \ldots, k\}$, using consistency we may suppose that $N=\{1, \ldots, k\} \cup\left\{h_{1}, \ldots, h_{k}\right\}$.

Recall that $\varphi_{l}\left(R_{l}^{\prime \prime}, R_{-l}\right)=1, \varphi_{1}\left(R_{l}^{\prime \prime}, R_{-l}\right)=l$, and $\varphi_{2}\left(R_{l}^{\prime \prime}, R_{-l}\right)=h \notin\{1, \ldots, k\}$. Now let $R_{l}^{\prime \prime \prime}$ : $h, 1, l, \ldots$ Now if $\varphi_{l}\left(R_{l}^{\prime \prime \prime}, R_{-l}\right)=h$, then let $\hat{R}_{l}: h, l, \ldots$ By strategy-proofness and non-bossiness, $\varphi\left(\hat{R}_{l}, R_{-l}\right)=\varphi\left(R_{l}^{\prime \prime \prime}, R_{-l}\right)$. Let $\varphi_{h}\left(\hat{R}_{l}, R_{-l}\right)=t$ and $\hat{R}_{l}^{\prime}: t, h, l, \ldots$ Note that $t \in\{2, \ldots, k\}$. If $\varphi_{l}\left(\hat{R}_{l}^{\prime}, R_{-l}\right)=h$, then by strategy-proofness and non-bossiness, $\varphi\left(\hat{R}_{l}^{\prime}, R_{-l}\right)=\varphi\left(\hat{R}_{l}, R_{-l}\right)$. Now this is a contradiction to Lemma 4 because the cycle in which $l$ and $h$ trade is not a top cycle for any strict transformation of $\left(\hat{R}_{l}^{\prime}, R_{-l}\right)$.

Thus, $\varphi_{l}\left(\hat{R}_{l}^{\prime}, R_{-l}\right)=t$ and $\varphi_{h}\left(\hat{R}_{l}^{\prime}, R_{-l}\right)=h$ (because $\left.R_{h}: t, h, \ldots\right)$. But now we can remove $h$ from the economy and $N \backslash\{h\}$ contains fewer than $2 k$ agents. But we may again apply the above conclusions to $N \backslash\{h\}$, a contradiction.

Thus, $\varphi_{l}\left(R_{l}^{\prime \prime \prime}, R_{-l}\right)=1$. By non-bossiness, $\varphi\left(R_{l}^{\prime \prime \prime}, R_{-l}\right)=\varphi\left(R_{l}^{\prime \prime}, R_{-l}\right)$. Let $R_{h}^{\prime}$ : $1, \varphi_{h}\left(R_{l}^{\prime \prime \prime}, R_{-l}\right), h, \ldots \quad$ Now if $\varphi_{h}\left(R_{l}^{\prime \prime \prime}, R_{h}^{\prime}, R_{-l, h}\right)=\varphi_{h}\left(R_{l}^{\prime \prime \prime}, R_{-l}\right)$, then by non-bossiness, $\varphi\left(R_{l}^{\prime \prime \prime}, R_{h}^{\prime}, R_{-l, h}\right)=\varphi\left(R_{l}^{\prime \prime \prime}, R_{-l}\right)$. But now by Lemma 2 , agent $h$ must trade before agents 1 and $l$ because $\operatorname{top}\left(R_{l}^{\prime \prime \prime}\right)=\{h\}$ and agent 1 must trade before agent $h$ because $\operatorname{top}\left(R_{h}^{\prime}\right)=\{1\}$. Obviously, this is impossible. Thus, $\varphi_{h}\left(R_{l}^{\prime \prime \prime}, R_{h}^{\prime}, R_{-l, h}\right)=1$. 
If $\varphi_{l}\left(R_{l}^{\prime \prime \prime}, R_{h}^{\prime}, R_{-l, h}\right)=l$, the we can remove $l$ from the economy and $N \backslash\{l\}$ contains fewer than $2 k$ agents. But we may again apply the above conclusions to $N \backslash\{l\}$, a contradiction. Similarly, if $\varphi_{l}\left(R_{l}^{\prime \prime \prime}, R_{h}^{\prime}, R_{-l, h}\right)=h$, then let $\hat{R}_{l}: h, l, \ldots$ and $\hat{R}_{h}: 1, h, \ldots$ Now by strategy-proofness and nonbossiness we obtain $\varphi\left(\hat{R}_{l}, \hat{R}_{h}, R_{-l, h}\right)=\varphi\left(R_{l}^{\prime \prime \prime}, R_{h}^{\prime}, R_{-l, h}\right)$. But now $\operatorname{top}\left(\hat{R}_{l}\right)=h$ and $h \notin\{1, \ldots, k\}$. Now as above for $\hat{R}_{l}: 1, h, l, \ldots$ we obtain $\varphi_{l}\left(\hat{R}_{l}^{\prime}, \hat{R}_{h}, R_{-l, h}\right)=1$ and $\varphi_{h}\left(\hat{R}_{l}^{\prime}, \hat{R}_{h}, R_{-l, h}\right)=h$. But now we can remove $h$ from the economy and $N \backslash\{h\}$ contains fewer than $2 k$ agents. But we may again apply the above conclusions to $N \backslash\{h\}$, a contradiction. This finishes the proof.

Using Lemma 5 we now show that $\varphi \sim f^{\succ}$.

Lemma 6. For all $R \in \mathcal{R}^{N}, \varphi(R) \sim^{R} f^{\succ}(R)$.

Proof. Let $R \in \mathcal{R}^{N}$ and let $c$ be a top cycle in $f^{\succ}(R)$ with trading coalition $C$. By Lemma 4 , $\varphi_{i}(R) I_{i} f_{i}^{\succ}(R)$ for all $i \in C$. By Lemma $5, \cup_{i \in C}\left\{\varphi_{i}(R)\right\}=C$. Thus, we may remove $C$ from the economy $R$. By consistency,

$$
\varphi_{i}\left(\left.R_{-C}\right|_{N \backslash C}\right)=\varphi_{i}(R)
$$

for all $i \in N \backslash C$.

Since $f^{\succ}$ satisfies consistency and $\cup_{i \in C}\left\{f_{i}^{\succ}(R)\right\}=C$, we also have

$$
f_{i}^{\succ}\left(\left.R_{-C}\right|_{N \backslash C}\right)=f_{i}^{\succ}(R)
$$

for all $i \in N \backslash C$.

Now let again $\hat{c}$ be a top cycle in $f^{\succ}\left(\left.R_{-C}\right|_{N \backslash C}\right)$ with trading coalition $\hat{C}$. Now by Lemma 4 , we have $\varphi_{i}\left(\left.R_{-C}\right|_{N \backslash C}\right) I_{i} f_{i}^{\succ}\left(\left.R_{-C}\right|_{N \backslash C}\right)$ for all $i \in \hat{C}$. Thus, by (2) and (3), $\varphi_{i}(R) I_{i} f_{i}^{\succ}(R)$ for all $i \in \hat{C}$.

Again by Lemma $5, \cup_{i \in \hat{C}}\left\{\varphi_{i}\left(\left.R_{-C}\right|_{N \backslash C}\right)\right\}=\hat{C}$. Thus, by (2) and $(3), \cup_{i \in \hat{C}}\left\{\varphi_{i}(R)\right\}=\hat{C}=$ $\cup_{i \in \hat{C}}\left\{f_{i}^{\succ}(R)\right\}$.

Now we may remove $C \cup \hat{C}$ from the economy $R$ and use the same argumentation as above. Because $N$ is finite, we obtain $\varphi_{i}(R) I_{i} f_{i}^{\succ}(R)$ for all $i \in N$. Thus, $\varphi(R) \sim^{R} f^{\succ}(R)$, the desired conclusion.

\section{Discussion}

Indifferences with the endowment: If indifferences with the endowment are allowed, then Theorem 1 is not true. The reason is that ties in indifference classes containing the endowment may be broken differently than ties in indifference classes not containing the endowment. More precisely, we may have for agent 1 two tie-breakers $\succ_{1}$ and $\tilde{\succ}_{1}$ such that for any indifference class $T$ with $1 \notin T$, ties are broken according to $\succ_{1}$ whereas for any indifference class $T$ with $1 \in T$, ties are broken according to $\tilde{\succ}_{1}$. 
More formally, let $\tilde{\mathcal{R}}_{i}^{N}$ denote the set of all weak preference relations on $N$ and let $\tilde{\mathcal{R}}^{N}=$ $\times_{i \in N} \tilde{\mathcal{R}}_{i}^{N}$. Given two profiles of fixed tie-breakers $\succ$ and $\tilde{\succ}$, we define $f^{\succ, \tilde{\succ}}$ as follows: for all $N \subseteq \mathcal{N}$ and all $R \in \mathcal{R}^{N}$, let $R^{\prime} \in S T(R)$ be such that for all $i \in N$, for any maximal indifference class $^{15} T \subseteq N$ in $R_{i}:\left.R_{i}^{\prime}\right|_{T}=\left.\succ_{i}\right|_{T}$ if $i \notin T$ and $\left.R_{i}^{\prime}\right|_{T}=\left.\tilde{\succ}_{i}\right|_{T}$ if $i \in T$. Then we set $f^{\succ, \tilde{\succ}}(R)=f\left(R^{\prime}\right)$.

Using the same arguments as in the proof of Theorem 1 and distinguishing between indifference classes containing the endowment and indifference classes not containing the endowment, we obtain the following variant of Theorem 1.

Theorem 2. Let indifferences with the endowment be allowed.

(a) For any two profiles of fixed tie-breakers $\succ$ and $\tilde{\succ}, f^{\succ, \tilde{\succ}}$ satisfies individual rationality, strategy-proofness, weak efficiency, non-bossiness, and consistency.

(b) If a rule $\varphi$ satisfies individual rationality, strategy-proofness, weak efficiency, non-bossiness, and consistency, then there exist two profiles $\succ$ and $\tilde{\succ}$ of fixed tie-breakers such that $\varphi \sim f^{\succ, \tilde{\succ}}$.

Domains: Instead of considering the full domain $\mathcal{R}^{\mathcal{N}}$ (and its full subdomains), we may consider any domain $\mathcal{D}^{\mathcal{N}}$ (and its subdomains) in between the full domain and the strict domain, i.e. such that $\mathcal{P}^{\mathcal{N}} \subseteq \mathcal{D}^{\mathcal{N}} \subseteq \mathcal{R}^{\mathcal{N}}$. Theorem 1 remains unchanged for any such domain $\mathcal{D}^{\mathcal{N}}$.

More formally, let $\mathcal{P}^{\mathcal{N}} \subseteq \mathcal{D}^{\mathcal{N}} \subseteq \mathcal{R}^{\mathcal{N}}$. For all $N \subseteq \mathcal{N}$, let $\mathcal{D}^{N}=\left\{\left.R_{N}\right|_{N}: R \in \mathcal{D}^{\mathcal{N}}\right\}$. Furthermore, for all $i \in \mathcal{N}$, let

$$
D_{i}=\left\{j \in \mathcal{N}: \text { for some } R_{i} \in \mathcal{D}_{i}^{\mathcal{N}} \text { and } k \in \mathcal{N} \backslash\{j\}, j I_{i} k P_{i} i\right\}
$$

denote the set of agents which agent $i$ ranks indifferent and above his endowment for some preference in the domain $\mathcal{D}_{i}^{\mathcal{N}}$.

Below we will just require that a tie-breaker $\succ_{i}$ is a strict and complete relation on $D_{i}$ and that if for some $R_{i} \in \mathcal{D}_{i}^{\mathcal{N}}$ and some triple $j, k, l \in D_{i}$ we have $j I_{i} k I_{i} l$, then $\left.\succ_{i}\right|_{\{j, k, l\}}$ is transitive.

Theorem 3. Let $\mathcal{P}^{\mathcal{N}} \subseteq \mathcal{D}^{\mathcal{N}} \subseteq \mathcal{R}^{\mathcal{N}}$.

(a) For any profile $\succ$ of fixed tie-breakers, $f^{\succ}$ satisfies individual rationality, strategy-proofness, weak efficiency, non-bossiness, and consistency on $\mathcal{D}^{\mathcal{N}}$ and its subdomains.

(b) If a rule $\varphi$ satisfies individual rationality, strategy-proofness, weak efficiency, non-bossiness, and consistency on $\mathcal{D}^{\mathcal{N}}$ and its subdomains, then there exists a profile $\succ$ of fixed tie-breakers such that $\varphi \sim f^{\succ}$.

Here note that in Lemma 3 we only need to show transitivity of $\succ_{i}$ for $j \succ_{i} k$ and $k \succ_{i} l$ if there is some preference $R_{i} \in \mathcal{D}_{i}^{\mathcal{N}}$ such that $j I_{i} k I_{i} l$. Then, of course, the proof of Lemma 3 is valid because $\left.R_{i}\right|_{\{i, j, k, l\}}$ is the preference used in that proof.

\footnotetext{
${ }^{15}$ The set $T \subseteq N$ is a maximal indifference class in $R_{i}$ if (i) for all $j, k \in T, j I_{i} k$ and (ii) for all $j \in T$ and all $k \in N \backslash T, j P_{i} k$ or $k P_{i} j$.
} 
If we never have $j I_{i} k I_{i} l$, then we never need to break a three-way tie. Now $\succ_{i}$ may not need to be transitive, i.e. we may have $j \succ_{i} k \succ_{i} l \succ_{i} j$. However, this does not create any problem because for any $R_{i}$, then we have at most one tie in the set $\{j, k, l\}$ (and the top trading cycle algorithm with fixed tie-breaking $f^{\succ}$ is well-defined).

Independence of the axioms:

Not individually rational: Fix a profile of tie-breakers $\succ$. For each $R$, break ties according to $\succ$ and apply serial dictatorship to the resulting $\succ(R)$ where agents with lower indices choose first.

Not strategy-proof: Fix a profile of tie-breakers $\succ$. For each $R$, break ties according to $\succ$. Then the agent with the lowest index (say 1 ) chooses the set of his most $\succ_{1}\left(R_{1}\right)$-preferred allocations which are individually rational and weakly efficient, say $M_{1}$. Observe that agent 1 receives the same object (say $l$ ) under all those allocations. Now let agent $l$ choose the set of his most $\succ_{l}\left(R_{l}\right)$-preferred allocations in $M_{1}$, and so on.

Not weakly efficient: Fix an allocation $\mu \in A_{\mathcal{N}}$ such that $\mu$ constitutes a cycle for $\mathcal{N}$. Now for all $N \subseteq \mathcal{N}$ and all $R \in \mathcal{R}^{N}$, (i) if $N=\mathcal{N}$ and $\mu$ is individually rational under $R$, then $\varphi(R)=\mu$, and (ii) otherwise $\varphi_{i}(R)=i$ for all $i \in N$.

Not non-bossy: Let $\mathcal{N}=\{1,2,3\}, \succ_{1}: 3,2,1, \succ_{2}: 1,3,2$, and $\succ_{3}: 1,2,3$. Let $\succ=\left(\succ_{1}, \succ_{2}, \succ_{3}\right)$. For all $N \subseteq \mathcal{N}$, (i) if $N=\mathcal{N}$ and both $2,3 \in \operatorname{top}\left(R_{1}\right), 3 P_{2} 2$ and $1 \in \operatorname{top}\left(R_{3}\right)$, then $\psi(R)=(2,3,1)$, and (ii) otherwise $\psi(R)=f^{\succ}(R)$. It is easy to check that $\psi$ satisfies individual rationality, strategyproofness, and weak efficiency. Furthermore, we cannot use consistency to reduce economies of the type (i) because $\psi(R)$ is a trading cycle for $\mathcal{N}$. Hence, $\psi$ satisfies consistency. Finally, $\psi$ violates non-bossiness because for the profile $R \in R^{\mathcal{N}}$ with

\begin{tabular}{c|c|c}
$R_{1}$ & $R_{2}$ & $R_{3}$ \\
\hline 2,3 & 1 & 1 \\
1 & 3 & 3 \\
& 2 & 2
\end{tabular}

we have $\psi(R)=(2,3,1)$. Let $R_{1}^{\prime}: 2,3,1$. Note that $\left(R_{1}^{\prime}, R_{2}, R_{3}\right) \in \mathcal{P}^{\mathcal{N}}$. Since $\psi$ satisfies individual rationality, strategy-proofness, weak efficiency, and consistency, from Lemma 1 we obtain $\psi\left(R_{1}^{\prime}, R_{-1}\right)=f\left(R_{1}^{\prime}, R_{-1}\right)=(2,1,3)$. Since $\psi_{1}\left(R_{1}^{\prime}, R_{-1}\right)=\psi_{1}(R)$ and $\psi\left(R_{1}^{\prime}, R_{-1}\right) \neq \psi(R), \psi$ violates non-bossiness.

Not consistent: Let $|\mathcal{N}| \geq 3$ and $\succ$ and $\succ^{\prime}$ be two distinct profiles of tie-breakers. For all $N \subseteq \mathcal{N}$ and all $R \in \mathcal{R}^{N}$, (i) if $|N|$ is even, then $\phi(R)=f^{\succ}(R)$, and (ii) if $|N|$ is odd, then $\phi(R), f^{\succ^{\prime}}(R)$. It is easy to verify that $\phi$ satisfies individual rationality, strategy-proofness, weak efficiency, and non-bossiness. Obviously, $\phi$ violates consistency. 
Remark 4. Regarding the last example, fix $N$ and for this remark, let $\varphi: \mathcal{R}^{N} \longrightarrow \mathcal{A}_{N}$ be a rule. Now one may wonder whether the following is true (for fixed $N$ ): if $\varphi$ satisfies individual rationality, strategy-proofness, weak efficiency, and non-bossiness, then there exists a profile of fixed tie-breakers such that $\left.\varphi \sim f^{\succ}\right|_{\mathcal{R}^{N}}$. The following example shows that (b) of Theorem 1 is not true for fixed $N$, i.e. consistency is necessary: Let $N=\{1,2,3,4\}$ and let $\succeq$ and $\succeq^{\prime}$ be two distinct profiles of tie-breakers. For any $R \in \mathcal{R}_{N}$, let $\tilde{\succ}(R) \in S T(R)$ be such that for all $i \in N$ : (i) if $R_{i}$ contains a three-way tie, then $\tilde{\succ}_{i}\left(R_{i}\right)=\succ_{i}\left(R_{i}\right)$, and (ii) otherwise $\tilde{\succ}_{i}\left(R_{i}\right)=\succ_{i}^{\prime}\left(R_{i}\right)$. For all $R \in \mathcal{R}^{N}$, let $\tilde{\varphi}(R)=f(\tilde{\succ}(R))$. Using the same arguments as in the proof of (a) of Theorem 1 it can be seen that $\tilde{\varphi}$ satisfies individual rationality, strategy-proofness, weak efficiency, and non-bossiness. ${ }^{16}$

\section{References}

Abdulkadiroğlu, A., and T. Sönmez (1998): Random Serial Dictatorship and the Core from Random Endowments in House Allocation Problems, Econometrica 66, 689-701.

Abdulkadiroğlu, A., and T. Sönmez (1999): House Allocation with Existing Tenants, Journal of Economic Theory 88, 233-260.

Abdulkadiroğlu, A., and T. Sönmez (2003): School Choice: a Mechanism Design Approach, American Economic Review 93, 729-747.

Abdulkadiroğlu, A., P. Pathak, and A.E. Roth (2009): Strategy-proofness versus Efficiency in Matching with Indifferences: Redesigning the NYC High School Match, American Economic Review 99, 1954-1978.

Alcalde-Unzu, J., and E. Molis (2011): Exchange of Indivisible Goods and Indifferences: the Top Trading Absorbing Sets Mechanisms, Games and Economic Behavior 73, 1-16.

Barberà, S., and M.O. Jackson (1995): Strategy-Proof Exchange, Econometrica 63, 51-87.

Bird, C.G. (1984): Group Incentive Compatibility in a Market with Indivisible Goods, Economics Letters 14, 309-313.

Bogomolnaia, A., R. Deb, and L. Ehlers (2005): Strategy-Proof Assignment on the Full Preference Domain, Journal of Economic Theory 123, 161-186.

Crawford, V.P. (1991): Comparative Statics in Matching Markets, Journal of Economic Theory $54,389-400$.

Ehlers, L. (2002): Coalitional Strategy-Proof House Allocation, Journal of Economic Theory 105, 298-317.

Ehlers, L. (2004): Monotonic and Implementable Solutions in Generalized Matching Problems, Journal of Economic Theory 114, 358-369.

\footnotetext{
${ }^{16}$ Note that $\tilde{\varphi}$ is not utility-equivalent to top-trading with fixed tie-breaking: for instance, let $2 \succ_{1} 3$ and $3 \succ_{1}^{\prime} 2$, and $\operatorname{top}\left(R_{2}\right)=\{1\}=\operatorname{top}\left(R_{3}\right)$ and $\operatorname{top}\left(R_{4}\right)=\{4\}$. Now if $\operatorname{top}\left(R_{1}\right)=\{2,3,4\}$, then $\tilde{\varphi}_{2}(R)=1$, and if $\operatorname{top}\left(R_{1}\right)=\{2,3\}$, then $\tilde{\varphi}_{3}(R)=1$.
} 
Ehlers, L., and B. Klaus (2007): Consistent House Allocation, Economic Theory 30, 561-574.

Ergin, H.I. (2002): Efficient Resource Allocation on the Basis of Priorities, Econometrica 70, 2489-2497.

Gale, D., and L. Shapley (1962): College Admissions and the Stability of Marriage, American Mathematical Monthly 69, 9-15.

Jaramillo, P., and V. Manjunath (2012): The Difference Indifference Makes in Strategy-Proof Allocation of Objects, Journal of Economic Theory, forthcoming.

Kesten, O. (2007): An Inventory of Top Trading Cycles Mechanisms for School Choice Problems, Working Paper.

Kesten, O. (2010): School Choice with Consent, Quarterly Journal of Economics 125, 1297-1348.

Klaus, B., F. Klijn, and M. Walzl (2010): Farsighted House Allocation, Journal of Mathematical Economics 46, 817-824.

Kojima, F., and M. Manea (2010): Axioms for Deferred Acceptance, Econometrica 78, 633-653.

Larsson, B., and L.-G. Svensson (2005): Strategy-Proofness, Core, and Sequential Trade, Review of Economic Design 9, 167-190.

Ma, J. (1994): Strategy-Proofness and the Strict Core in a Market with Indivisibilities, International Journal of Game Theory 23, 75-83.

Miyagawa, E. (2001): House Allocation with Transfers, Journal of Economic Theory 100, 329-355.

Miyagawa, E. (2002): Strategy-Proofness and the Core in House Allocation Problems, Games and Economic Behavior 38, 347-361.

Pápai, S. (2000): Strategyproof Assignment by Hierarchical Exchange, Econometrica 68, 14031433.

Pycia, M., and M.U. Ünver (2009): A Theory of House Allocation and Exchange Mechanisms, Working Paper.

Quint, T., and J. Wako (2004): On Houseswapping, the Strict Core, Segmentation, and Linear Programming, Mathematics of Operations Research 29, 861-877.

Roth, A.E. (1982): Incentive Compatibility in a Market with Indivisible Goods, Economics Letters 9, 127-132.

Roth, A.E., and A. Postlewaite (1977): Weak versus Strong Domination in a Market with Indivisible Goods, Journal of Mathematical Economics 4, 131-137.

Satterthwaite, M.A., and H. Sonnenschein (1981): Strategy-Proof Allocation Mechanisms at Differentiable Points, Review of Economic Studies 48, 587-597.

Shapley, L, and H. Scarf (1974): On Cores and Indivisibility, Journal of Mathematical Economics $1,23-28$.

Sönmez, T. (1999): Strategy-Proofness and Essentially Single-Valued Cores, Econometrica 67, $677-689$.

Sönmez, T., and M.U. Ünver (2009): Matching, Allocation, and Exchange of Discrete Resources, 
Jess Benhabib, Alberto Bisin, and Matthew Jackson (eds.) Handbook of Social Economics, Elsevier, forthcoming.

Sönmez, T., and M.U. Ünver (2010): House Allocation with Existing Tenants: A Characterization, Games and Economic Behavior 69, 425-445

Svensson, L.-G. (1994): Queue allocation of indivisible goods, Social Choice and Welfare 11, $323-330$.

Svensson, L.-G. (1999): Strategy-Proof Allocation of Indivisible Goods, Social Choice and Welfare $16,557-567$.

Thomson, W. (1992): Consistency in Exchange Economies, Working Paper.

Thomson, W. (2009): Consistent Allocation Rules, book manuscript.

J. Wako (1984): A Note on the Strong Core of a Market with Indivisible Goods, Journal of Mathematical Economics 13, 189-194. 